\title{
Actions infinitésimales dans la correspondance de Langlands locale $p$-adique
}

\author{
Gabriel Dospinescu *
}

20 novembre 2018

\begin{abstract}
Résumé
Let $V$ be a two-dimensional absolutely irreducible $\overline{\mathbf{Q}_{p}}$-representation of $\operatorname{Gal}\left(\overline{\mathbf{Q}_{p}} / \mathbf{Q}_{p}\right)$ and let $\Pi(V)$ be the $\mathrm{GL}_{2}\left(\mathbf{Q}_{p}\right)$ Banach representation associated by Colmez's $p$-adic Langlands correspondence. We establish a link between the action of the Lie algebra of $\mathrm{GL}_{2}\left(\mathbf{Q}_{p}\right)$ on the locally analytic vectors $\Pi(V)^{\text {an }}$ of $\Pi(V)$, the connection $\nabla$ on the $(\varphi, \Gamma)$-module associated to $V$ and the Sen polynomial of $V$. This answers a question of Harris, concerning the infinitesimal character of $\Pi(V)^{\text {an }}$. Using this result, we give a new proof of a theorem of Colmez, stating that $\Pi(V)$ has nonzero locally algebraic vectors if and only if $V$ is potentially semi-stable with distinct Hodge-Tate weights.
\end{abstract}

\section{Introduction}

Cet article s'inscrit dans le cadre de la correspondance de Langlands locale $p$-adique, imaginée par Breuil [5] (au moins dans le cas potentiellement semi-stable) et établie par Colmez [10] pour $\mathrm{GL}_{2}\left(\mathbf{Q}_{p}\right)$, à la suite des travaux de Colmez [11], Berger-Breuil [3], Kisin [28]. Cette correspondance encode la correspondance de Langlands locale classique pour $\mathrm{GL}_{2}\left(\mathbf{Q}_{p}\right)$.

Soit $L$ une extension finie de $\mathbf{Q}_{p}$ et soit $V$ une $L$-représentation absolument irréductible de $\operatorname{Gal}\left(\overline{\mathbf{Q}_{p}} / \mathbf{Q}_{p}\right)$, de dimension 2. En utilisant la théorie des $(\varphi, \Gamma)$-modules de Fontaine [23, Colmez associe à $V$ une $\mathrm{GL}_{2}\left(\mathbf{Q}_{p}\right)$-représentation de Banach $\Pi(V)$, unitaire, admissible, topologiquement irréductible et décrit les vecteurs localement analytiques $\Pi(V)^{\text {an }}$ de $\Pi(V)$ en fonction de $V$. Cependant, la description est très indirecte, ce qui rend l'étude de $\Pi(V)^{\text {an }}$ un peu délicate : par exemple, on ne sait pas si $\Pi(V)^{\text {an }}$ est toujours de longueur finie. Dans cet article, on calcule l'action infinitésimale de $\mathrm{GL}_{2}\left(\mathbf{Q}_{p}\right)$ sur $\Pi(V)^{\text {an }}$, et on en déduit une nouvelle démonstration d'un résultat de Colmez caractérisant les représentations $V$ telles que $\Pi(V)$ possède des vecteurs localement algébriques (c'est ce résultat qui permet de faire le pont avec la correspondance classique). Il y a d'autres problèmes auxquels les techniques de cet article s'appliquent, le cas le plus notable étant (voir [12] et [15]) des conjectures de Berger, Breuil [3] et Emerton [18], qui décrivent de manière précise l'espace $\Pi(V)^{\text {an }}$ quand $V$ est trianguline.

\footnotetext{
${ }^{*}$ C.M.L.S, Ecole Polytechnique, gabriel.dospinescu@math.polytechnique.fr
} 


\subsection{Notations}

On fixe dans toute la suite un nombre premier $p$, une extension finie $L$ de $\mathbf{Q}_{p}$ et une clôture algébrique $\overline{\mathbf{Q}_{p}}$ de $\mathbf{Q}_{p}$, contenant $L$. Soit $\chi: \operatorname{Gal}\left(\overline{\mathbf{Q}_{p}} / \mathbf{Q}_{p}\right) \rightarrow \mathbf{Z}_{p}^{*}$ le caractère cyclotomique; alors $\chi$ induit un isomorphisme de $\Gamma=\operatorname{Gal}\left(\mathbf{Q}_{p}\left(\mu_{p \infty}\right) / \mathbf{Q}_{p}\right)$ sur $\mathbf{Z}_{p}^{*}$, et on note $a \mapsto \sigma_{a}$ l'isomorphisme réciproque de $\mathbf{Z}_{p}^{*}$ sur $\Gamma$ (on a donc $\sigma_{a}(\zeta)=\zeta^{a}$ pour tout $\left.\zeta \in \mu_{p^{\infty}}\right)$. Soient $\mathscr{R}$ l'anneau de Robba (c'est l'anneau des séries de Laurent $\sum_{n \in \mathbf{Z}} a_{n} T^{n}$ à coefficients dans $L$, qui convergent sur une couronne du type $0<v_{p}(T) \leq r$, où $r$ dépend de la série), $\mathscr{E}^{\dagger}$ le sous-anneau de $\mathscr{R}$ des éléments bornés (c'est un corps) et $\mathscr{E}$ le complété de $\mathscr{E}^{\dagger}$ pour la valuation $p$-adique. On munit ces anneaux d'actions continues de $\Gamma$ et d'un Frobenius $\varphi$, commutant entre elles, en posant $\varphi(T)=(1+T)^{p}-1$ et $\sigma_{a}(T)=(1+T)^{a}-1$ si $a \in \mathbf{Z}_{p}^{*}$.

\subsection{Le faisceau $\Delta \rightarrow \Delta \otimes U$}

Une $L$-représentation de $\operatorname{Gal}\left(\overline{\mathbf{Q}_{p}} / \mathbf{Q}_{p}\right)$ est un $L$-espace vectoriel $V$ de dimension finie, muni d'une action $L$-linéaire continue de $\operatorname{Gal}\left(\overline{\mathbf{Q}_{p}} / \mathbf{Q}_{p}\right)$. Soit $V$ une $L$-représentation de dimension 2 de $\operatorname{Gal}\left(\overline{\mathbf{Q}_{p}} / \mathbf{Q}_{p}\right)$, absolument irréductible. La théorie de Fontaine [23], complétée par des travaux de Cherbonnier-Colmez [6], Berger [2] et Kedlaya [27], associe à $V$ des modules $D=D(V), D^{\dagger}, D_{\text {rig }}$, libres de rang 2 sur $\mathscr{E}$, $\mathscr{E}^{\dagger}$ et $\mathscr{R}$, munis d'une action semi-linéaire de $\Gamma$ et d'un Frobenius $\varphi$ semi-linéaire, commutant à $\Gamma$ (le module $D^{\dagger}$ n'a pas d'intérêt propre, mais il permet de passer de $D$ à $D_{\text {rig }}$ et vice-versa). Enfin, on dispose sur $\Delta \in\left\{D, D_{\text {rig }}\right\}$ d'un inverse à gauche $\psi$ de $\varphi$ qui commute à $\Gamma$.

On peut utiliser ces opérateurs pour définir une action 1$]$ du monoïde $P^{+}=\left(\begin{array}{cc}\mathbf{z}_{p}-\{0\} & \mathbf{z}_{p} \\ 0 & 1\end{array}\right)$ sur $\Delta$, en posant

$$
\left(\begin{array}{cc}
p^{k} a & b \\
0 & 1
\end{array}\right) z=(1+T)^{b} \cdot \varphi^{k}\left(\sigma_{a}(z)\right)
$$

pour $z \in \Delta$ et $k \geq 0, a \in \mathbf{Z}_{p}^{*}, b \in \mathbf{Z}_{p}$. Le monoïde $P^{+}$agit aussi sur $\mathbf{Z}_{p}$, par $\left(\begin{array}{ll}a & b \\ 0 & 1\end{array}\right) x=$ $a x+b$. Colmez définit un faisceau $U \rightarrow \Delta \otimes U$ sur $\mathbf{Z}_{p}$, équivariant sous l'action de $P^{+}$, dont les sections sur $a+p^{n} \mathbf{Z}_{p}$ sont

$$
\Delta \otimes\left(a+p^{n} \mathbf{Z}_{p}\right)=(1+T)^{a} \cdot \varphi^{n}(\Delta) \subset \Delta
$$

(en particulier $\Delta \otimes \mathbf{Z}_{p}=\Delta$ ) et la restriction $\operatorname{Res}_{a+p^{n}} \mathbf{Z}_{p}: \Delta=\Delta \otimes \mathbf{Z}_{p} \rightarrow \Delta \otimes\left(a+p^{n} \mathbf{Z}_{p}\right)$ est

$$
\operatorname{Res}_{a+p^{n}} \mathbf{Z}_{p}=\left(\begin{array}{cc}
1 & a \\
0 & 1
\end{array}\right) \circ \varphi^{n} \circ \psi^{n} \circ\left(\begin{array}{cc}
1 & -a \\
0 & 1
\end{array}\right) .
$$

Faisons agir $\mathrm{GL}_{2}\left(\mathbf{Q}_{p}\right)$ sur $\mathbf{P}^{1}\left(\mathbf{Q}_{p}\right)$ par $\left(\begin{array}{ll}a & b \\ c & d\end{array}\right) z=\frac{a z+b}{c z+d}$ (la restriction de cette action à $P^{+}$est celle définie ci-dessus). Un des résultats principaux de [10] est le fait que le faisceau précédent sur $\mathbf{Z}_{p}$ s'étend en un faisceau $\operatorname{sur} \mathbf{P}^{1}\left(\mathbf{Q}_{p}\right)$, équivariant sous l'action de $\mathrm{GL}_{2}\left(\mathbf{Q}_{p}\right)$ (les formules pour l'action de $\mathrm{GL}_{2}\left(\mathbf{Q}_{p}\right)$ sont nettement plus compliquées que celles ci-dessus, mais sont en fait inutiles pour les applications). Soit $w=\left(\begin{array}{ll}0 & 1 \\ 1 & 0\end{array}\right)$. Comme

\footnotetext{
${ }^{1}$ Voir la remarque 3.2 pour l'origine et la signification de ces formules.
} 
$\mathbf{P}^{1}\left(\mathbf{Q}_{p}\right)$ est obtenu par recollement à partir de $\mathbf{Z}_{p}$ et $w \cdot \mathbf{Z}_{p}$ le long de $\mathbf{Z}_{p}^{*}$, on peut décrire l'espace des sections globales du faisceau attaché à $\Delta$ par

$$
\Delta \otimes \mathbf{P}^{1}=\left\{\left(z_{1}, z_{2}\right) \in \Delta \times \Delta \mid \quad \operatorname{Res}_{\mathbf{Z}_{p}^{*}}\left(z_{2}\right)=w_{D}\left(\operatorname{Res}_{\mathbf{Z}_{p}^{*}}\left(z_{1}\right)\right)\right\},
$$

où $w_{D}$ est la restriction de $w$ à $\Delta \mathbf{Q} \mathbf{Z}_{p}^{*}$. Soit $\delta_{D}=\chi^{-1} \cdot \operatorname{det} V$, que l'on voit comme caractère de $\mathbf{Q}_{p}^{*}$ par la théorie locale du corps de classes2 2 . La représentation $\Pi(V)$ attachée à $V$ par la correspondance de Langlands locale $p$-adique vit alors dans une suite exacte

$$
0 \rightarrow \Pi(V)^{*} \otimes\left(\delta_{D} \text { odet }\right) \rightarrow D \otimes \mathbf{P}^{1} \rightarrow \Pi(V) \rightarrow 0
$$

de $\mathrm{GL}_{2}\left(\mathbf{Q}_{p}\right)$-modules topologiques. Ses vecteurs localement analytiques vivent dans une suite exacte analogue

$$
0 \rightarrow\left(\Pi(V)^{\text {an }}\right)^{*} \otimes\left(\delta_{D} \circ \text { det }\right) \rightarrow D_{\text {rig }} \otimes \mathbf{P}^{1} \rightarrow \Pi(V)^{\text {an }} \rightarrow 0 .
$$

Par ailleurs, si $U$ est un ouvert compact de $\mathbf{P}^{1}\left(\mathbf{Q}_{p}\right)$, on dispose d'une application de prolongement par 0 de $\Delta \otimes U$ dans $\Delta \otimes \mathbf{P}^{1}$, ce qui permet en particulier de voir $D_{\text {rig }}=$ $D_{\text {rig }} \otimes \mathbf{Z}_{p}$ comme un sous-module de $D_{\text {rig }} \otimes \mathbf{P}^{1}$.

\subsection{Actions infinitésimales}

Dans [10], Colmez montre que $D_{\text {rig }} \otimes \mathbf{P}^{1}$ est un module sous l'action 3 de l'algèbre enveloppante $U\left(\mathfrak{g l}_{2}\right)$ de Lie $\left(\mathrm{GL}_{2}\left(\mathbf{Q}_{p}\right)\right)$, et cette action stabilise $D_{\text {rig }} \otimes U$ pour tout ouvert compact $U$ de $\mathbf{P}^{1}\left(\mathbf{Q}_{p}\right)$; en particulier, cette action stabilise le sous-module $D_{\text {rig }}=D_{\text {rig }} \otimes \mathbf{Z}_{p}$. Soit

$$
h=\left(\begin{array}{cc}
1 & 0 \\
0 & -1
\end{array}\right), \quad u^{+}=\left(\begin{array}{ll}
0 & 1 \\
0 & 0
\end{array}\right), \quad u^{-}=\left(\begin{array}{ll}
0 & 0 \\
1 & 0
\end{array}\right)
$$

la base usuelle de $\mathfrak{s l}_{2}$, de sorte que $\mathrm{I}_{2}=\left(\begin{array}{ll}1 & 0 \\ 0 & 1\end{array}\right), u^{+}, u^{-}, h$ forment une base de $\mathfrak{g l}_{2}$. D'après Berger [2], $D_{\text {rig }}$ est aussi muni d'une action infinitésimale de $\Gamma$, à travers la connexion

$$
\nabla(z)=\lim _{\gamma \rightarrow 1} \frac{\gamma(z)-z}{\chi(\gamma)-1} .
$$

Enfin, rappelons que $t=\log (1+T) \in \mathscr{R}$ et que, grâce aux travaux de Sen [32], on peut associer à $V$ un polynôme $P_{\mathrm{Sen}, \mathrm{V}} \in L[X]$ de degré 2 , unitaire, dont les racines s'appellent les poids de Hodge-Tate généralisés de $V$. Si $V$ est Hodge-Tate, il s'agit des poids de Hodge-Tate classiques de $V$ (avec la convention que le poids de $\mathbf{Q}_{p}(1)$ est 1 ).

Théorème 1.1. Soit $V$ une L-représentation absolument irréductible de $\operatorname{Gal}\left(\overline{\mathbf{Q}_{p}} / \mathbf{Q}_{p}\right)$, de dimension 2 , à poids de Hodge-Tate $a, b$, de telle sorte que $P_{\mathrm{Sen}, \mathrm{V}}=(X-a)(X-b)$. $L$ 'action de $\mathfrak{g l}_{2}$ sur $D_{\text {rig }}$ est donnée par $\mathrm{I}_{2}(z)=(a+b-1) z$,

$$
u^{-}(z)=-\frac{P_{\text {Sen }, \mathrm{V}}(\nabla)(z)}{t}, \quad h(z)=2 \nabla(z)-(a+b-1) z, \quad u^{+}(z)=t z .
$$

\footnotetext{
${ }^{2}$ Donc $\chi$, vu comme caractère de $\mathbf{Q}_{p}^{*}$ est $x \rightarrow x \cdot|x|_{p}$, où $|\cdot|_{p}$ est la valeur absolue $p$-adique.

${ }^{3}$ Plus précisément, il démontre que $D_{\text {rig }} \otimes \mathbf{P}^{1}$ est un module sous l'action de l'algèbre des distributions d'un sous-groupe ouvert compact suffisament petit de $\mathrm{GL}_{2}\left(\mathbf{Q}_{p}\right)$.
} 
On déduit du théorème 1.1 l'action de $U\left(\mathfrak{g l}_{2}\right)$ sur $D_{\text {rig }} \otimes \mathbf{P}^{1}$, car un élément de $D_{\text {rig }} \otimes \mathbf{P}^{1}$ est de la forme $z_{1}+w \cdot z_{2}$ avec $z_{1}, z_{2} \in D_{\text {rig. }}$ La preuve du théorème 1.1 fait jouer un rôle essentiel à l'élément de Casimir

$$
C=u^{+} u^{-}+u^{-} u^{+}+\frac{1}{2} h^{2} \in U\left(\mathfrak{s l}_{2}\right),
$$

qui engendre le centre de l'algèbre $\mathrm{U}\left(\mathfrak{s l}_{2}\right)$. En effet, il n'est pas difficile de vérifier que $C$ induit un endomorphisme du $(\varphi, \Gamma)$-module $D_{\text {rig. }}$. Des propriétés standard des $(\varphi, \Gamma)$ modules entraînent que cet endomorphisme est scalaire. Pour identifier ce scalaire, on utilise la théorie de Hodge $p$-adique, en particulier les techniques différentielles de Berger et Fontaine (voir la partie 2.5). Le théorème 1.1 est une conséquence facile du résultat suivant, qui répond aussi à une question de Harris ([18], remark 3.3.8).

Théorème 1.2. Si $V$ est comme dans le théorème 1.1, l'action de $C$ sur $D_{\text {rig }} \otimes \mathbf{P}^{1}$, et donc sur $\Pi(V)^{\mathrm{an}}$, est la multiplication par $\frac{(b-a)^{2}-1}{2}$.

En fait, les deux théorèmes précédents sont équivalents à l'énoncé suivant, portant sur l'involution $w_{D}$ de Colmez.

Corollaire 1.3. Si $z \in D_{\text {rig }} \otimes \mathbf{Z}_{p}^{*}$, on a

$$
w_{D}(t z)=-\frac{P_{\mathrm{Sen}, \mathrm{V}}(\nabla)\left(w_{D}(z)\right)}{t} .
$$

Si $a, b \in L$, l'espace des représentations $V$ dont les poids de Hodge-Tate généralisés sont $a, b$ est une variété de dimension 3. Les représentations de Banach attachées à ces représentations galoisiennes ont le même caractère infinitésimal d'après le théorème 1.2, mais elles sont deux à deux non isomorphes (la correspondance de Langlands locale $p$-adique étant injective, d'après un théorème de Colmez). On voit ainsi qu'il y a une infinité de représentations de Banach de $\mathrm{GL}_{2}\left(\mathbf{Q}_{p}\right)$, absolument irréductibles, unitaires, admissibles, ayant le même caractère infinitésimal. Cela ne se produit pas dans la théorie classique des représentations unitaires des groupes réels (semi-simples, mais oublions cela pour un moment), d'après des résultats classiques (mais profonds) de Harish-Chandra.

Le théorème 1.2 couplé à un théorème récent et très délicat de Paškūnas [30], qui démontre la surjectivité de la correspondance $V \rightarrow \Pi(V)$, donne le résultat suivant :

Théorème 1.4. Soit $p>3$ et soit $\Pi$ une L-représentation de Banach de $\mathrm{GL}_{2}\left(\mathbf{Q}_{p}\right)$, unitaire, admissible, absolument irréductible. Alors $\Pi^{\text {an }}$ admet un caractère infinitésimal.

L'hypothèse $p>3$ apparaît dans les travaux de Paškūnas ; il est raisonnable de penser que le résultat reste vrai pour $p \leq 3$ (cf. [17] pour des résultats dans cette direction). Paškūnas démontre 4 en outre l'analogue du lemme de Schur : si П est comme dans le théorème [1.4, alors $\operatorname{End}_{L\left[\mathrm{GL}_{2}\left(\mathbf{Q}_{\mathrm{p}}\right)\right]}^{\mathrm{cont}}(\Pi)=L$. On peut se demander si le même résultat est valable en remplaçant $\Pi$ par $\Pi^{\text {an }}$. Cela démontrerait le théorème 1.4 de manière plus

\footnotetext{
${ }^{4}$ Sa preuve est très détournée, mais une preuve plus simple d'un résultat plus général est donnée dans [16. Elle repose sur une version du lemme de Quillen due à Ardakov et Wadsley [1].
} 
directe (mais pas le théorème 1.2), mais je ne sais pas le faire. Je ne sais pas non plus si $\Pi^{\text {an }}$ est de longueur finie pour une représentation $\Pi$ comme dans le théorème 1.4, même s'il est très probable que la réponse soit positivf 5 . Notons que dans la théorie des groupes réels, ce résultat est une conséquence formelle du fait qu'il y a seulement un nombre fini de (classes de) représentations unitaires irréductibles ayant un caractère infinitésimal donné, mais comme on l'a remarqué ces résultats ne sont plus valables en $p$-adique.

La motivation principale du théorème 1.1 était de donner une preuve "analytique" du théorème suivant, dû à Colmez ([10], thm. VI.6.13, VI.6.18).

Théorème 1.5. Si $V$ est comme dans le théorème 1 1.1, alors $\Pi(V)$ a des vecteurs localement algébriques non nuls si et seulement si $V$ est potentiellement semi-stable à poids de Hodge-Tate distincts.

Notons que le théorème 1.5 joue un rôle crucial dans la preuve de la conjecture de Fontaine-Mazur en dimension 2 (sous certaines hypothèses faibles) par Emerton [19]. Il joue aussi un rôle important dans la preuve par Kisin [29] de la même conjecture (mais Kisin a besoin d'informations plus fines concernant les vecteurs localement algébriques).

Notre démonstration du théorème 1.5 est nettement plus directe que celle que donne Colmez dans [10], qui repose sur deux généralisations des lois de réciprocité explicites de Kato [26] et Perrin-Riou [31], 8]. Il est assez amusant de constater que même si on utilise beaucoup des résultats de Colmez, ce sont précisément ces résultats qui ne sont pas utilisés dans sa preuve du théorème 1.5.

\subsection{Remerciements}

Ce travail est une partie de ma thèse de doctorat, réalisée sous la direction de Pierre Colmez et de Gaëtan Chenevier. Je leur suis profondément réconnaissant pour tout ce qu'ils m'ont appris. Il sera plus qu'évident au lecteur combien cet article doit au travail monumental [10] de Colmez. Mais plus encore, je lui suis profondément reconnaissant pour les nombreuses et longues discussions que nous avons eues autour de cet article. En particulier, sans son observation que l'action de $\mathfrak{g l}_{2}$ se prolonge au module de Fontaine, cet article n'aurais jamais vu le jour. Je remercie vivement Wang Shanwen pour des discussions éclairantes autour de certains points de l'article, ainsi que le rapporteur, dont les remarques m'ont beaucoup aidé à améliorer la présentation.

\section{$2 \quad$ Théorie de Hodge $p$-adique et $(\varphi, \Gamma)$-modules}

Le but de ce chapitre est de rappeler quelques résultats standard concernant les liens entre la théorie des $(\varphi, \Gamma)$-modules et la théorie de Hodge $p$-adique. Ces résultats seront

\footnotetext{
${ }^{5}$ Voir aussi la conjecture faite dans [16] et ce que ce l'on sait pour l'instant concernant cette question. Le cas où $V$ est trianguline est démontré dans [12, où $\Pi(V)^{\text {an }}$ est étudié en détail, répondant ainsi à des conjectures de Berger, Breuil et Emerton. L'ingrédient principal de la preuve est l'étude du module de Jacquet analytique de $\Pi(V)^{\text {an }}$, qui peut se faire facilement [15] en utilisant les résultats de cet article.
} 
pleinement utilisés dans les chapitres 4 et 5, mais la proposition 2.14 sera suffisante pour la démonstration du théorème 1.1. Pour expliquer certaines constructions classiques, il faut malheureusement ouvrir la boîte de Pandore des anneaux de Fontaine. Le lecteur pourra facilement faire abstraction de la plupart de ces anneaux, car pour la suite seuls les anneaux $\mathbf{B}_{\mathrm{dR}}, \tilde{\mathbf{B}}, \tilde{\mathbf{B}}^{+}$et $\mathscr{R}$ (voir plus bas pour les définitions) seront utilisés. Voir [22] et [13] pour les preuves des assertions concernant les anneaux de Fontaine, ainsi que [14] pour une vue d'ensemble. Soit $\varepsilon^{(n)}$ une racine primitive d'ordre $p^{n}$ de l'unité, telle que $\left(\varepsilon^{(n+1)}\right)^{p}=\varepsilon^{(n)}$ pour tout $n$. On pose $F_{n}=\mathbf{Q}_{p}\left(\varepsilon^{(n)}\right), L_{n}=L \otimes_{\mathbf{Q}_{p}} F_{n}$ et $L_{\infty}=\cup_{n} L_{n}$.

\subsection{Anneaux de fonctions analytiques}

Notons, pour $n \geq 1$,

$$
r_{n}=\frac{1}{p^{n-1}(p-1)}=v_{p}\left(\varepsilon^{(n)}-1\right) .
$$

Définition 2.1. a) Soit $\mathscr{E}$ l'anneau des séries de Laurent $\sum_{k \in \mathbf{Z}} a_{k} T^{k}$, avec $\left(a_{k}\right)_{k \in \mathbf{Z}}$ une suite bornée d'éléments de $L$ et $\lim _{k \rightarrow-\infty} a_{k}=0$.

b) Soit $\mathscr{E}^{\left(0, r_{n}\right]}$ l'anneau des séries $f=\sum_{k \in \mathbf{Z}} a_{k} T^{k} \in \mathscr{E}$ qui convergent sur la couronne $0<v_{p}(T) \leq r_{n}$. Soit $\mathscr{E}^{\dagger}$ la réunion des $\mathscr{E}^{\left(0, r_{n}\right]}$.

c) Soit $\left.\mathscr{E}] 0, r_{n}\right]$ l'anneau des séries $\sum_{k \in \mathbf{Z}} a_{k} T^{k}$ qui convergent sur la couronne $0<$ $v_{p}(T) \leq r_{n}$ et soit $\mathscr{R}$ l'anneau de Robba, réunion des $\left.\left.\mathscr{E}\right] 0, r_{n}\right]$ à l'intérieur de $L\left[\left[T, T^{-1}\right]\right]$.

Tous ces anneaux sont munis de topologies naturelles, ainsi que d'une action continue de $\Gamma$, définie par $\sigma_{a}(f)(T)=f\left((1+T)^{a}-1\right)$ pour $a \in \mathbf{Z}_{p}^{*}$. Les anneaux $\mathscr{E}$ et $\mathscr{R}$ sont aussi munis d'un Frobenius continu $\varphi$, défini par $\varphi(f)(T)=f\left((1+T)^{p}-1\right)$ et commutant à l'action de $\Gamma$. Si $\Lambda \in\{\mathscr{E}, \mathscr{R}\}$, tout élément $f \in \Lambda$ s'écrit de manière unique sous la forme

$$
f=\sum_{i=0}^{p-1}(1+T)^{i} \varphi\left(f_{i}\right),
$$

avec $f_{i} \in \Lambda$. En posant $\psi(f)=f_{0}$, on obtient un endomorphisme continu de $L$-espaces vectoriels $\psi: \Lambda \rightarrow \Lambda$ tel que $\psi(\varphi(f))=f$ pour tout $f \in \Lambda$.

Soit $t=\log (1+T) \in \mathscr{R}$. On a alors $\sigma_{a}(t)=$ at pour tout $a \in \mathbf{Z}_{p}^{*}$ et $\varphi(t)=p t$. De plus, on dispose pour tout $n \geq 1$ d'une injection $\Gamma$-équivariante $\left.\left.\varphi^{-n}: \mathscr{E}^{[}\right] 0, r_{n}\right] \rightarrow L_{n}[[t]]$, qui envoie $f$ sur $f\left(\varepsilon^{(n)} e^{t / p^{n}}-1\right)$ (noter que, comme $f$ converge en $\varepsilon^{(n)}-1, f\left(\varepsilon^{(n)} e^{t / p^{n}}-1\right.$ ) est bien défini en tant qu'élément de $\left.L_{n}[[t]]\right)$.

\subsection{Anneaux de Fontaine}

Soit $\mathbf{C}_{p}$ le complété de $\overline{\mathbf{Q}_{p}}$ et soit $O_{\mathbf{C}_{p}}$ l'anneau de ses entiers. Soit $v_{p}$ la valuation $p$-adique sur $\mathbf{C}_{p}$. On note $\tilde{\mathbf{E}}^{+}$l'anneau des suites $x=\left(x^{(n)}\right)_{n \geq 0} \in O_{\mathbf{C}_{p}}^{\mathbf{N}}$ telles que $\left(x^{(n+1)}\right)^{p}=x^{(n)}$

\footnotetext{
${ }^{6} \mathscr{E}^{\left.] 0, r_{n}\right]}$ est muni de la topologie de Fréchet déduite des normes sup sur les couronnes $r_{m} \leq v_{p}(T) \leq r_{n}$, avec $m \geq n$ et $\mathscr{R}=\cup_{n} \mathscr{E}^{\left[0, r_{n}\right]}$ est muni de la topologie limite inductive. Soit $O_{\mathscr{E}}$ le sous-anneau de $\mathscr{E}$ formé des séries $\sum_{n \in \mathbf{Z}} a_{n} T^{n}$ avec $a_{n} \in O_{L}$. On munit $O_{\mathscr{E}}$ de la topologie dont une base de voisinages de 0 est donnée par les $T^{n} O_{L}[[T]]+p^{k} O_{\mathscr{E}}$ et on met la topologie limite inductive sur $\mathscr{E}=\cup_{n} p^{-n} O_{\mathscr{E}}$.
} 
pour tout $n$, l'addition étant définie par

$$
(x+y)^{(n)}=\lim _{j \rightarrow \infty}\left(x^{(n+j)}+y^{(n+j)}\right)^{p^{j}}
$$

et la multiplication étant définie composante par composante; c'est un anneau parfait de caractéristique $p$, muni d'une action $\sqrt{7}$ de $\operatorname{Gal}\left(\overline{\mathbf{Q}_{p}} / \mathbf{Q}_{p}\right)$, commutant au Frobenius $x \rightarrow x^{p}$, que l'on note $\varphi$. En posant $v_{E}(x)=v_{p}\left(x^{(0)}\right)$ pour $x \in \tilde{\mathbf{E}}^{+}$, on obtient une valuation sur l'anneau $\tilde{\mathbf{E}}^{+}$. L'élément $\bar{T}=\left(\varepsilon^{(n)}\right)_{n}-1 \in \tilde{\mathbf{E}}^{+}$satisfait $v_{E}(\bar{T})=\frac{p}{p-1}$ et le corps des fractions $\tilde{\mathbf{E}}=\tilde{\mathbf{E}}^{+}[1 / \bar{T}]$ de $\tilde{\mathbf{E}}^{+}$est algébriquement clos et complet pour la valuation $v_{E}$.

Soit $\tilde{\mathbf{A}}^{+}=W\left(\tilde{\mathbf{E}}^{+}\right)$(resp. $\left.\tilde{\mathbf{A}}=W(\tilde{\mathbf{E}})\right)$ l'anneau des vecteurs de Witt à coefficients dans $\tilde{\mathbf{E}}^{+}($resp. $\tilde{\mathbf{E}})$ et soit $\tilde{\mathbf{B}}^{+}=\tilde{\mathbf{A}}^{+}[1 / p]($ resp. $\tilde{\mathbf{B}}=\tilde{\mathbf{A}}[1 / p])$. Tout élément de $\tilde{\mathbf{A}}^{+}$(resp. $\tilde{\mathbf{A}})$ s'écrit de manière unique sous la forme $\sum_{k \geq 0} p^{k}\left[x_{k}\right]$, avec $x_{k} \in \tilde{\mathbf{E}}^{+}$(resp. $\tilde{\mathbf{E}}$ ). Ici $[x] \in \tilde{\mathbf{A}}^{+}$(resp. $\left.\tilde{\mathbf{A}}\right)$ est le représentant de Teichmuller de $x \in \tilde{\mathbf{E}}^{+}$(resp. $\left.\tilde{\mathbf{E}}\right)$. Le Frobenius et l'action de $\operatorname{Gal}\left(\overline{\mathbf{Q}_{p}} / \mathbf{Q}_{p}\right)$ sur $\tilde{\mathbf{E}}^{+}$et $\tilde{\mathbf{E}}$ induisent un Frobenius bijectif $\varphi$ et une action de $\operatorname{Gal}\left(\overline{\mathbf{Q}_{p}} / \mathbf{Q}_{p}\right)$ sur $\tilde{\mathbf{A}}^{+}, \tilde{\mathbf{A}}, \tilde{\mathbf{B}}$ et $\tilde{\mathbf{B}}^{+}$. Le résultat suivant, dans lequel $T=[1+\bar{T}]-1 \in \tilde{\mathbf{A}}^{+}$, est standard ([22], propositions 2.4, 2.12 et 2.17).

Proposition 2.2. L'application $\theta: \tilde{\mathbf{A}}^{+} \rightarrow O_{\mathbf{C}_{p}}$ définie par $\theta(x)=\sum_{n \geq 0} p^{n} x_{n}^{(0)}$ si $x=$ $\sum_{n \geq 0} p^{n}\left[x_{n}\right]$ est un morphisme surjectif d'anneaux et $\operatorname{Ker}(\theta)=\omega \cdot \tilde{\mathbf{A}}^{+}$, où $\omega=\frac{T}{\varphi^{-1}(T)}$. Le séparé complété $\mathbf{B}_{\mathrm{dR}}^{+}$de $\tilde{\mathbf{B}}^{+}$pour la topologie $\omega$-adique est un anneau de valuation discrète, d'uniformisante $\omega$ ot

$$
t=\log (1+T)=\sum_{n \geq 1}(-1)^{n-1} \frac{T^{n}}{n} .
$$

Le morphisme $\theta: \tilde{\mathbf{B}}^{+} \rightarrow \mathbf{C}_{p}$ s'étend par continuité en un morphisme surjectif $\mathbf{B}_{\mathrm{dR}}^{+} \rightarrow$ $\mathbf{C}_{p}$, dont le noyau est engendré par $\omega$ ou $t$. On note $\mathbf{B}_{\mathrm{dR}}=\mathbf{B}_{\mathrm{dR}}^{+}[1 / t]$, qui est donc un corps de valuation discrète, d'uniformisante $t$ et de corps résiduel $\mathbf{C}_{p}$.

On dispose d'une myriade de sous-anneaux de $\tilde{\mathbf{B}}$, qui sont stables sous l'action de $\operatorname{Gal}\left(\overline{\mathbf{Q}_{p}} / \mathbf{Q}_{p}\right)$ et font le lien entre la théorie de Hodge $p$-adique et la théorie des $(\varphi, \Gamma)$ modules.

a) Pour tout $r>0$, soit $\tilde{\mathbf{B}}^{(0, r]}$ l'ensemble des $x=\sum_{k>>-\infty} p^{k}\left[x_{k}\right] \in \tilde{\mathbf{B}}$ tels que $\lim _{k \rightarrow \infty} v_{E}\left(x_{k}\right)+\frac{k}{r}=\infty$. Soit $\tilde{\mathbf{B}}^{\dagger}$ la réunion des $\tilde{\mathbf{B}}^{(0, r]}$ à l'intérieur de $\tilde{\mathbf{B}}$. Comme $\varphi\left(\tilde{\mathbf{B}}^{(0, r]}\right)=\tilde{\mathbf{B}}^{(0, r / p]}, \tilde{\mathbf{B}}^{\dagger}$ est stable sous l'action de $\varphi$, qui est bijectif.

b) Si $x=\sum_{k>>-\infty} p^{k}\left[x_{k}\right] \in \tilde{\mathbf{B}}^{(0, r]}$, on note

$$
v^{(0, r]}(x)=\inf _{k \in \mathbf{Z}}\left(v_{E}\left(x_{k}\right)+\frac{k}{r}\right) .
$$

Soit $\tilde{\mathbf{B}}^{[0, r]}$ la complétion de $\tilde{\mathbf{B}}^{(0, r]}$ pour la topologie de Fréchet induite par les semivaluations $\left(\min \left(v^{(0, s]}, v^{(0, r]}\right)\right)_{0<s \leq r}$ et soit $\tilde{\mathbf{B}}_{\text {rig }}$ la réunion des $\tilde{\mathbf{B}}^{] 0, r]}$. Cet anneau est muni d'un Frobenius bijectif, obtenu par prolongement à partir du Frobenius sur $\tilde{\mathbf{B}}^{\dagger}$.

\footnotetext{
${ }^{7}$ Cette action est induite par l'action tautologique de $\operatorname{Gal}\left(\overline{\mathbf{Q}_{p}} / \mathbf{Q}_{p}\right)$ sur $O_{\mathbf{C}_{p}}$.

${ }^{8}$ Noter que l'on utilise la même lettre que pour l'élément $t=\log (1+T)$ de $\mathscr{R}$. Cela s'explique par le fait que l'on a une injection naturelle de $\mathscr{R}^{+}=\mathscr{R} \cap L[[T]]$ dans $\mathbf{B}_{\mathrm{dR}}^{+}$, qui envoie $f$ sur $f\left(e^{t}-1\right)$ et cette injection envoie l'élément $t$ de $\mathscr{R}$ sur $t \in \mathbf{B}_{\mathrm{dR}}^{+}$.
} 
c) Soit $\mathbf{A}_{\mathbf{Q}_{p}}$ l'anneau 9 des séries de Laurent $\sum_{k \in \mathbf{Z}} a_{k} T^{k}$, où $a_{k} \in \mathbf{Z}_{p}$ et $\lim _{k \rightarrow-\infty} a_{k}=$ 0 . On voit $\mathbf{A}_{\mathbf{Q}_{p}}$ à l'intérieur de $\tilde{\mathbf{A}}$ en envoyant $T$ sur $[1+\bar{T}]-1$. Soit $\mathbf{B}$ l'adhérence, pour la topologie $p$-adique, de l'extension maximale non-ramifiée de $\mathbf{A}_{\mathbf{Q}_{p}}[1 / p]$ dans $\tilde{\mathbf{B}}$. Ce sous-anneau de $\tilde{\mathbf{B}}$ est stable sous l'action de $\varphi$. Finalement, on note $\mathbf{B}^{(0, r]}=\mathbf{B} \cap \tilde{\mathbf{B}}^{(0, r]}$ et $\mathbf{B}_{\text {rig }}=\mathscr{R} \otimes_{\mathscr{E}^{\dagger}} \mathbf{B}^{\dagger}$.

On peut montrer 10 que l'on a des identifications compatibles aux actions 11 de $\varphi$ et $\Gamma$

$$
\left(L \otimes \mathbf{Q}_{p} \mathbf{B}\right)^{H}=\mathscr{E}, \quad\left(L \otimes \mathbf{Q}_{p} \mathbf{B}^{\dagger}\right)^{H}=\mathscr{E}^{\dagger}, \quad\left(L \otimes \mathbf{Q}_{p} \mathbf{B}_{\text {rig }}\right)^{H}=\mathscr{R}
$$

et $\left(L \otimes \mathbf{Q}_{p} \mathbf{B}^{(0, r]}\right)^{H}=\mathscr{E}^{(0, r]}$ pour tout $r>0$ assez petit.

\section{$2.3(\varphi, \Gamma)$-modules}

Définition 2.3. Un $(\varphi, \Gamma)$-module $D$ sur $\Lambda \in\left\{\mathscr{E}, \mathscr{E}^{\dagger}, \mathscr{R}\right\}$ est (dans cet article) un $\Lambda$ module libre de type fini, muni d'une action continue semi-linéaire de $\Gamma$ et d'un opérateur semi-linéaire $\varphi$, commutant à $\Gamma$ et tel que $\Lambda \varphi(D)=D$.

Dans la suite de ce chapitre, on fixe une L-représentation $V$ de $\operatorname{Gal}\left(\overline{\mathbf{Q}_{p}} / \mathbf{Q}_{p}\right)$. La théorie de Fontaine [23], raffinée par les travaux de Cherbonnier-Colmez [6], Kedlaya [27] et Berger [2], associe à $V$ une famille de $\Gamma$ et $(\varphi, \Gamma)$-modules.

Définition 2.4. On définit:

a) Les $(\varphi, \Gamma)$-modules $D=\left(\mathbf{B} \otimes_{\mathbf{Q}_{p}} V\right)^{H}, D^{\dagger}=\left(\mathbf{B}^{\dagger} \otimes_{\mathbf{Q}_{p}} V\right)^{H}, \tilde{D}_{\text {rig }}=\left(\tilde{\mathbf{B}}_{\text {rig }} \otimes_{\mathbf{Q}_{p}} V\right)^{H}$,

$$
D_{\text {rig }}=\left(\mathbf{B}_{\text {rig }} \otimes_{\mathbf{Q}_{p}} V\right)^{H}=\mathscr{R} \otimes_{\mathscr{E}^{\dagger}} D^{\dagger}
$$

$\operatorname{sur} \mathscr{E}, \mathscr{E}^{\dagger},\left(L \otimes \mathbf{Q}_{p} \tilde{\mathbf{B}}_{\text {rig }}\right)^{H}$ et $\mathscr{R}$, respectivement.

b) Les $\Gamma$-modules $D^{\left(0, r_{n}\right]}=\left(\mathbf{B}^{\left(0, p^{-n}\right]} \otimes_{\mathbf{Q}_{p}} V\right)^{H}, \tilde{D}^{\left(0, r_{n}\right]}=\left(\tilde{\mathbf{B}}^{\left(0, p^{-n}\right]} \otimes \mathbf{Q}_{p} V\right)^{H}, D^{\left.0, r_{n}\right]}=$ $\left.\left.\mathscr{E}^{\mathscr{E}}\right] 0, r_{n}\right] \otimes_{\mathscr{E}\left(0, r_{n}\right]} D^{\left(0, r_{n}\right]}$ et $\tilde{D}^{\left[0, r_{n}\right]}=\left(\tilde{\mathbf{B}}^{\left.] 0, p^{-n}\right]} \otimes_{\mathbf{Q}_{p}} V\right)^{H}$.

c) Les $(\varphi, \Gamma)$-modules $\tilde{D}=\left(\tilde{\mathbf{B}} \otimes \mathbf{Q}_{p} V\right)^{H}, \tilde{D}^{+}=\left(\tilde{\mathbf{B}}^{+} \otimes \mathbf{Q}_{p} V\right)^{H}$.

d) Les $\Gamma$-modules $\tilde{D}_{\text {dif }}=\left(\mathbf{B}_{\mathrm{dR}} \otimes_{\mathbf{Q}_{p}} V\right)^{H}$ et $\tilde{D}_{\text {dif }}^{+}=\left(\mathbf{B}_{\mathrm{dR}}^{+} \otimes_{\mathbf{Q}_{p}} V\right)^{H}$.

On dispose alors du résultat fondamental suivant, dû à Cherbonnier et Colmez [6] .

Théorème 2.5. Il existe un entier $m(D)$ tel que $D^{\left(0, r_{m(D)}\right]}$ soit un $\mathscr{E}^{\left(0, r_{m(D)}\right]}$-module libre de rang $\operatorname{dim}_{L} V$, tel qu盘 $D=\mathscr{E} \otimes D^{\left(0, r_{m(D)}\right]}$. De plus, pour tout $n \geq m(D)$ on a $D^{\left(0, r_{n}\right]}=\mathscr{E}\left(0, r_{n}\right] \otimes D^{\left(0, r_{m(D)}\right]}$.

Remarque 2.6. 1) On déduit du théorème 2.5 que $D^{\left[0, r_{n}\right]}$, $D^{\dagger}$ et $D_{\text {rig }}$ s'obtiennent à partir de $D^{\left(0, r_{m(D)}\right]}$ par extension des scalaires à $\mathscr{E}^{\left[0, r_{n}\right]}, \mathscr{E}^{\dagger}$ et $\mathscr{R}$, respectivement. On obtient aussi un isomorphisme canonique $D=\mathscr{E} \otimes_{\mathscr{E}} D^{\dagger}$.

\footnotetext{
${ }^{9}$ Noter que $\mathbf{A}_{\mathbf{Q}_{p}}$ n'est rien d'autre que l'anneau $O_{\mathscr{E}}$ pour $L=\mathbf{Q}_{p}$.

${ }^{10} \mathrm{~L}$ 'action de $\operatorname{Gal}\left(\overline{\mathbf{Q}_{p}} / \mathbf{Q}_{p}\right)$ sur tous les anneaux et objets qui suivent est $L$-linéaire. Pour la preuve de ces isomorphismes, voir [13, propositions 7.1, 7.5 et 7.6, ainsi que [2, proposition 3.15.

${ }^{11}$ Noter que les actions de $\varphi$ et $\Gamma$ sur $T=[1+\bar{T}]-1$ sont données par $\varphi(T)=(1+T)^{p}-1$ et $\sigma_{a}(T)=(1+T)^{a}-1$, i.e. ce sont les mêmes que celles définies dans la partie 2.1 .

${ }^{12}$ Tous les produits tensoriels sont pris au-dessus de $\mathscr{E}^{\left(0, r_{m(D)}\right)}$.
} 
2) Dans la suite on se permettra d'augmenter un nombre fini de fois $m(D)$ si besoin est. Par exemple, dans le chapitre $\mathrm{V}$ de [10] on construit des entiers $m(D)$, $m_{1}(D), \ldots, m_{5}(D)$. On supposera que notre $m(D)$ est plus grand que toutes ces constantes. Remarque 2.7. On peut récupérer $V$ de manière fonctorielle à partir de $D, D^{\dagger}$ et $D_{\text {rig }}$ par

$$
V=\left(\left(L \otimes_{\mathbf{Q}_{p}} \mathbf{B}\right) \otimes_{\mathscr{E}} D\right)^{\varphi=1}=\left(\left(L \otimes_{\mathbf{Q}_{p}} \mathbf{B}^{\dagger}\right) \otimes_{\mathscr{E} \dagger} D^{\dagger}\right)^{\varphi=1}=\left(\left(L \otimes_{\mathbf{Q}_{p}} \tilde{\mathbf{B}}_{\text {rig }}\right) \otimes_{\mathscr{R}} D_{\text {rig }}\right)^{\varphi=1} .
$$

Comme on a aussi un isomorphisme fonctoriel $D_{\text {rig }}=\left(\mathbf{B}_{\text {rig }} \otimes_{\mathbf{Q}_{p}} V\right)^{H}$, le foncteur $V \rightarrow$ $D_{\text {rig }}$ est pleinement fidèle; en particulier $\operatorname{End}_{L\left[\operatorname{Gal}\left(\overline{\mathbf{Q}_{p}} / \mathbf{Q}_{p}\right)\right]}(V)=\operatorname{End}_{\varphi, \Gamma, \mathscr{R}}\left(D_{\text {rig }}\right)$ pour toute $L$-représentation $V$.

On finit cette partie en rappelant la construction des applications de localisation pour un $(\varphi, \Gamma)$-module. Elles vont jouer un rôle crucial dans la suite. Si $x=\sum_{k>>-\infty} p^{k}\left[x_{k}\right] \in$ $\tilde{\mathbf{B}}^{(0,1]}$, la série $\sum_{k>>-\infty} p^{k}\left[x_{k}\right]$ converge dans $\mathbf{B}_{\mathrm{dR}}^{+}$, ce qui fournit un morphisme naturel $\tilde{\mathbf{B}}^{(0,1]} \rightarrow \mathbf{B}_{\mathrm{dR}}^{+}$, qui se trouve être injectif. Ce morphisme s'étend en un morphisme, toujours injectif, de $\tilde{\mathbf{B}}^{[0,1]}$ dans $\mathbf{B}_{\mathrm{dR}}^{+}$. Composé avec l'isomorphisme $\tilde{\mathbf{B}}^{] 0,1]} \simeq \tilde{\mathbf{B}}^{\left[0, p^{-n}\right.}$ ] induit par $\varphi^{n}$, ce morphisme induit une application de localisation $\varphi^{-n}: \tilde{\mathbf{B}}^{\left.00, p^{-n}\right]} \rightarrow \mathbf{B}_{\mathrm{dR}}^{+}$, compatible avec l'action de $\operatorname{Gal}\left(\overline{\mathbf{Q}_{p}} / \mathbf{Q}_{p}\right)$.

Définition 2.8. On note $\varphi^{-n}: D^{\left[0, r_{n}\right]} \rightarrow \tilde{D}_{\text {dif }}^{+}$la composée de l'injection $D^{\left[0, r_{n}\right]} \subset$ $\tilde{D}^{\left[0, r_{n}\right]}=\left(\tilde{\mathbf{B}}^{\left[0, p^{-n}\right]} \otimes \mathbf{Q}_{p} V\right)^{H}$ avec l'application $\varphi^{-n} \otimes 1: \tilde{\mathbf{B}}^{\left[0, p^{-n}\right]} \otimes V \rightarrow \mathbf{B}_{\mathrm{dR}}^{+} \otimes V$. Cette injection $L$-linéaire, $\Gamma$-équivariante est appelée morphisme de localisation en $\varepsilon^{(n)}-1$.

Exemple 2.9. Si $D$ est le $(\varphi, \Gamma)$-module attaché à la représentation triviale, le morphisme de localisation est l'injection $\varphi^{-n}$ de $\mathscr{E}^{\mathbb{E}}{ }_{\left.0, r_{n}\right]} \rightarrow L_{n}[[t]]$ de la partie 2.1, composée avec l'inclusion $L_{n}[[t]] \subset L \otimes \mathbf{Q}_{p}\left(\mathbf{B}_{\mathrm{dR}}^{+}\right)^{H}$.

\subsection{Le module de Fontaine $D_{\mathrm{dif}, n}^{+}$}

Définition 2.10. Soit $D_{\mathrm{dif}, n}^{+}$le sous $L_{n}[[t]]$-module de $\tilde{D}_{\text {dif }}^{+}$engendré par l'image du morphisme de localisation $\varphi^{-n}: D^{\left.] 0, r_{n}\right]} \rightarrow \tilde{D}_{\mathrm{dif}}^{+}=\left(\mathbf{B}_{\mathrm{dR}}^{+} \otimes_{\mathbf{Q}_{p}} V\right)^{H}$.

Remarque 2.11. a) Pour $n \geq m(D)$ on a

$$
D_{\mathrm{dif}, n}^{+}=L_{n}[[t]] \otimes_{\left.\mathscr{E}] 0, r_{n}\right]} D^{\left.0, r_{n}\right]},
$$

où $L_{n}[[t]]$ est vu comme $\mathscr{E}^{\mathscr{E}}\left[0, r_{n}\right]$-module via $\varphi^{-n}$ (voir l'exemple 2.9). Le théorème 2.5 et la remarque 2.6 entraînent que $D_{\text {dif,n }}^{+}$est un $L_{n}[[t]]$-module libre de rang $\operatorname{dim}_{L} V$, stable sous l'action de $\Gamma$. De plus, $\Gamma_{n}$ agit de manière $L_{n}$-linéaire sur $D_{\mathrm{dif}, n}^{+}$.

b) La représentation $V$ est dite de de Rham si le $L$-espace vectoriel

$$
D_{\mathrm{dR}}(V)=\left(\mathbf{B}_{\mathrm{dR}} \otimes_{\mathbf{Q}_{p}} V\right)^{\mathrm{Gal}\left(\overline{\mathbf{Q}_{p}} / \mathbf{Q}_{p}\right)}
$$

est de $\operatorname{dimension} \operatorname{dim}_{L} V$. La filtration sur $D_{\mathrm{dR}}(V)$ est définie par $\operatorname{Fil}^{i}\left(D_{\mathrm{dR}}(V)\right)=$ $\left(t^{i} \mathbf{B}_{\mathrm{dR}}^{+} \otimes \mathbf{Q}_{p} V\right)^{\mathrm{Gal}\left(\overline{\mathbf{Q}_{p}} / \mathbf{Q}_{p}\right)}$ pour tout $i \in \mathbf{Z}$. On note aussi $D_{\mathrm{dR}}^{+}(V)=\operatorname{Fil}^{0}\left(D_{\mathrm{dR}}(V)\right)$. 
Si $V$ est de de Rham, alors on peut retrouver $D_{\mathrm{dR}}(V)$ et sa filtration à partir de $D_{\text {dif }, n}^{+}$par la recette $\operatorname{Fil}^{i}\left(D_{\mathrm{dR}}(V)\right)=\left(t^{i} D_{\text {dif }, n}^{+}\right)^{\Gamma}$, pour $n \geq m(D)$ et $i \in \mathbf{Z}$. De plus, $D_{\text {dif }, n}^{+}[1 / t]=L_{n}((t)) \otimes_{L} D_{\mathrm{dR}}(V)$ pour tout $n \geq m(D)$. Notons qu'avec nos conventions, si $V$ est de de Rham, alors les poids de Hodge-Tate de $V$ sont les opposés des sauts de la filtration sur $D_{\mathrm{dR}}(V)$.

c) Dans tous les cas, $D_{\mathrm{dif}, n}^{+}$engendre $\mathbf{B}_{\mathrm{dR}}^{+} \otimes_{\mathbf{Q}_{p}} V$ comme $\mathbf{B}_{\mathrm{dR}}^{+}$-module (voir [24]).

\subsection{L'action infinitésimale de $\Gamma$}

D'après Berger [2], lemme 4.1 et ce qui suit, l'action infinitésimale de $\Gamma$ sur $D_{\text {rig }}$ définit une connexion

$$
\nabla: D_{\text {rig }} \rightarrow D_{\text {rig }}, \quad \nabla(z)=\lim _{a \rightarrow 1} \frac{\sigma_{a}(z)-z}{a-1},
$$

qui commute à $\varphi$ et $\Gamma$ et laisse stable $D^{\left[0, r_{n}\right]}$ pour $n \geq m(D)$. Par exemple, si $V$ est triviale, alors $D_{\text {rig }}=\mathscr{R}$ et

$$
\nabla(f)=(1+T) \log (1+T) \cdot \frac{d}{d T} f(T)=t \cdot \frac{d f}{d t}
$$

pour $f \in \mathscr{R}$. En général, la connexion $\nabla$ satisfait $\nabla(f z)=\nabla(f) z+f \nabla(z)$ pour tout $z \in D_{\text {rig }}$ et tout $f \in \mathscr{R}$.

L'action de $\Gamma$ sur $D_{\text {dif, } n}^{+}$(avec $n \geq m(D)$ ) peut aussi se dériver, ce qui induit une connexion $L_{n}$-linéaire $\nabla=\lim _{a \rightarrow 1} \frac{\sigma_{a}-1}{a-1}$ sur $D_{\text {dif }, n}^{+}$, au-dessus de la connexion $t \frac{d}{d t}$ sur $L_{n}[[t]]$. Cette connexion préserve donc $t^{i} D_{\mathrm{dif}, n}^{+}$pour tout $i \geq 0$.

Définition 2.12. Les poids de Hodge-Tate généralisés (resp. le polynôme $P_{\text {Sen,V }}$ de Sen) de $V$ sont les valeurs propres (resp. le polynôme caractéristique) de $\nabla$ agissant sur le $L_{n}$-module libre de type fini $D_{\mathrm{Sen}, n}:=D_{\mathrm{dif}, n}^{+} / t D_{\mathrm{dif}, n}^{+}$.

Remarque 2.13. La définition 2.12 est compatible avec la définition classique13 des poids de Hodge-Tate généralisés. En effet, $\theta:\left(\mathbf{B}_{\mathrm{dR}}^{+} \otimes_{\mathbf{Q}_{p}} V\right)^{H} \rightarrow\left(\mathbf{C}_{p} \otimes_{\mathbf{Q}_{p}} V\right)^{H}$ identifie $D_{\text {Sen }, n}$ à un sous- $L_{n}$-module de $D_{\text {Sen }}(V)$, tel que $D_{\text {Sen }, n} \otimes_{L_{n}} L_{\infty}=D_{\text {Sen }}(V)$.

Le résultat suivant joue un rôle crucial dans la preuve du théorème 1.1.

Proposition 2.14. Soit $V$ une L-représentation de $\operatorname{Gal}\left(\overline{\mathbf{Q}_{p}} / \mathbf{Q}_{p}\right)$ et soit $D_{\text {rig }}$ le $(\varphi, \Gamma)$ module sur $\mathscr{R}$ attaché à $V$. Alors $P_{\mathrm{Sen}, \mathrm{V}}(\nabla)$ envoie $D_{\text {rig }}$ dans $t \cdot D_{\text {rig }}$.

Démonstration. Si $z \in D_{\text {rig }}$ et $\varphi^{-n}(z) \in t D_{\text {dif,n }}^{+}$pour tout $n$ assez grand, alors $z \in t D_{\text {rig }}$ (voir [2], lemmes 5.1 et 5.4). On se ramène donc à montrer que pour tout $z \in D_{\text {rig }}$ et pour tout $n$ assez grand on a $\varphi^{-n}\left(P_{\mathrm{Sen}, \mathrm{V}}(\nabla)(z)\right) \in t \cdot D_{\text {dif }, n}^{+}$. Puisque $\varphi^{-n}$ commute à $\nabla$, il suffit de vérifier que $P_{\mathrm{Sen}, \mathrm{V}}(\nabla)$ envoie $D_{\mathrm{dif}, \mathrm{n}}^{+}$dans $t \cdot D_{\mathrm{dif}, n}^{+}$. Cela découle du théorème de Cayley-Hamilton dans le $L_{n}$-module libre de type fini $D_{\mathrm{Sen}, n}$.

\footnotetext{
${ }^{13}$ D'après Sen [32], il existe un plus grand sous- $L_{\infty}$-module libre de type fini $D_{\text {Sen }}(V)$ de $\left(\mathbf{C}_{p} \otimes \mathbf{Q}_{p} V\right)^{H}$, qui est stable par $\Gamma$. Le module $D_{\text {Sen }}(V)$ engendre $\mathbf{C}_{p} \otimes_{\mathbf{Q}_{p}} V$ sur $\mathbf{C}_{p}$ et est de $\operatorname{rang} \operatorname{dim}_{L} V$. L'action infinitésimale de $\Gamma$ sur $D_{\text {Sen }}(V)$ définit un opérateur $L_{\infty}$-linéaire $\Theta_{\text {Sen }}$, dont les valeurs propres s'appelent les poids de Hodge-Tate généralisés de $V$.
} 


\subsection{Traces de Tate normalisées}

Soit $L_{\infty}=\cup_{n} L_{n}$. Pour tout $n \geq 0$ on dispose d'un projecteur $\Gamma$-équivariant $T_{n}: L_{\infty} \rightarrow$ $L_{n}$, défini par

$$
T_{n}(x)=\frac{1}{p^{j}} \operatorname{Tr}_{L_{n+j} / L_{n}}(x)
$$

pour tout $j$ tel que $x \in L_{n+j}$. On en déduit des projecteurs $\Gamma$-équivariants

$$
\operatorname{Res}_{p^{-n}} \mathbf{Z}_{p}: L_{\infty}((t)) \rightarrow L((t)), \quad \operatorname{Res}_{p^{-n}} \mathbf{Z}_{p}\left(\sum_{l>>-\infty} a_{l} t^{l}\right)=\sum_{l>>-\infty} T_{n}\left(a_{l}\right) t^{l} .
$$

Dans [8], proposition V.4.5, Colmez étend ces projecteurs en des projecteurs $L((t))$ linéaires continus (appelés traces de Tate normalisées) $\operatorname{Res}_{p^{-n}} \mathbf{Z}_{p}: L \otimes \mathbf{B}_{\mathrm{dR}}^{H} \rightarrow L_{n}((t))$, envoyant $L \otimes\left(\mathbf{B}_{\mathrm{dR}}^{+}\right)^{H}$ dans $L_{n}[[t]]$.

\subsection{L'opérateur $\psi$}

Comme $\varphi(D)$ engendre $D$, tout élément $z$ de $D$ s'écrit de manière unique sous la forme

$$
z=\sum_{i=0}^{p-1}(1+T)^{i} \varphi\left(z_{i}\right),
$$

avec $z_{i} \in D$, ce qui permet de définir un opérateur continu $\psi$ sur $D$ par $\psi(z)=z_{0}$. On vérifie facilement que $\psi(f \varphi(z))=\psi(f) z$ et $\psi(\varphi(f) z)=f \psi(z)$ pour $f \in \mathscr{E}$ et $z \in D$. De plus, $\psi$ commute à $\Gamma$. Tous ces résultats restent valables si on remplace $D$ par $D_{\text {rig }}$ et $\mathscr{E}$ par $\mathscr{R}$.

\section{Actions infinitésimales sur $D_{\text {rig }}$}

Dans ce chapitre on démontre le théorème 1.1. L'idée de la preuve est très simple : on montre que l'opérateur de Casimir (qui engendre le centre de l'algèbre $U\left(\mathfrak{s l}_{2}\right)$ ) induit un endomorphisme de $D_{\text {rig }}$, qui est forcément scalaire par des résultats de Berger et Kedlaya. Pour identifier ce scalaire, on utilise le lien entre la théorie de Hodge $p$-adique et les $(\varphi, \Gamma)$-modules, plus précisément la proposition 2.14. Pour en déduire l'action de $u^{-}$ sur $D_{\text {rig }}$ il ne reste plus qu'à revenir aux formules explicites donnant l'action de $\mathrm{GL}_{2}\left(\mathbf{Q}_{p}\right)$ et à exprimer l'action du Casimir seulement en fonction de $u^{-}$. Les premières parties de ce chapitre sont préliminaires et rappellent des constructions diverses et variées de Colmez. Dans la suite $V$ est une L-représentation absolument irréductible, de dimension 2 de $\operatorname{Gal}\left(\overline{\mathbf{Q}_{p}} / \mathbf{Q}_{p}\right)$. On note $D$ et $D_{\text {rig }}$ les $(\varphi, \Gamma)$-modules sur $\mathscr{E}$ et $\mathscr{R}$ attachés à $V$ (définition 2.4).

\subsection{Le faisceau $U \rightarrow \Delta \otimes U$}

Soit $\Delta \in\left\{D, D_{\text {rig }}\right\}$. Dans la partie 1.2, on a expliqué la construction d'un faisceau $P^{+}$ équivariant sur $\mathbf{Z}_{p}$, attaché à $\Delta$, qui se prolonge (d'après les chapitres II et $\mathrm{V}$ de [10]) 
en un faisceau sur $\mathbf{P}^{1}\left(\mathbf{Q}_{p}\right)$, équivariant sous l'action de $\mathrm{GL}_{2}\left(\mathbf{Q}_{p}\right)$. Soit $w_{D}$ la restriction à $\Delta \nabla \mathbf{Z}_{p}^{*}$ de l'action de l'involution $w=\left(\begin{array}{ll}0 & 1 \\ 1 & 0\end{array}\right)$, de telle sorte que l'espace des sections globales du faisceau attaché à $\Delta$ est

$$
\Delta \otimes \mathbf{P}^{1}=\left\{\left(z_{1}, z_{2}\right) \in \Delta \times \Delta \mid \quad \operatorname{Res}_{\mathbf{Z}_{p}^{*}}\left(z_{2}\right)=w_{D}\left(\operatorname{Res}_{\mathbf{Z}_{p}^{*}}\left(z_{1}\right)\right)\right\} .
$$

L'application de prolongement par zéro $i_{\mathbf{Z}_{p}}: \Delta \rightarrow \Delta \otimes \mathbf{P}^{1}$ définie par $i_{\mathbf{Z}_{p}}(z)=$ $\left(z, w_{D}\left(\operatorname{Res}_{\mathbf{Z}_{p}^{*}}(z)\right)\right)$ permet d'identifier $\Delta$ à un sous-module de $\Delta \nabla \mathbf{P}^{1}$, ce que l'on fera sans plus de commentaire. Tout élément $z$ de $\Delta \otimes \mathbf{P}^{1}$ peut alors s'écrire $z=z_{1}+w \cdot z_{2}$ avec $z_{1}, z_{2} \in \Delta$. Rappelons que $\delta_{D}=\chi^{-1} \cdot \operatorname{det} V$ est vu comme caractère de $\mathbf{Q}_{p}^{*}$. L'action de $\mathrm{GL}_{2}\left(\mathbf{Q}_{p}\right)$ sur $\Delta=\Delta \otimes \mathbf{Z}_{p}$ est donnée par les formules suivantes :

a) $\left(\begin{array}{ll}a & 0 \\ 0 & a\end{array}\right) z=\delta_{D}(a) z$ et $\left(\begin{array}{ll}p & 0 \\ 0 & 1\end{array}\right) z=\varphi(z)$.

b) Si $a \in \mathbf{Z}_{p}^{*}$, alors $\left(\begin{array}{cc}a & 0 \\ 0 & 1\end{array}\right) z=\sigma_{a}(z)$.

c) Pour tout $b \in p \mathbf{Z}_{p}$ on a $\left(\begin{array}{ll}1 & b \\ 0 & 1\end{array}\right) z=(1+T)^{b} \cdot z$ et

$$
\left(\begin{array}{cc}
1 & 0 \\
b & 1
\end{array}\right) z=w_{D}\left((1+T)^{b} \cdot w_{D}\left(\operatorname{Res}_{\mathbf{Z}_{p}^{*}}(z)\right)\right)+u_{b}\left(\operatorname{Res}_{p} \mathbf{Z}_{p}(z)\right)
$$

aver14, pour $x \in \varphi(D)$,

$$
u_{b}(x)=\delta_{D}^{-1}(1-b)(1+T)^{\frac{1}{b-1}} w_{D}\left[(1+T)^{b(1-b)} \sigma_{(1-b)^{2}}\left(w_{D}((1+T) x)\right)\right] .
$$

Remarque 3.1. Les formules donnant l'action du Borel supérieur sur $\Delta=\Delta \nabla \mathbf{Z}_{p}$ sont facile à manipuler et expliquent les formules simples pour l'action de $\mathrm{I}_{2}, u^{+}$et $h$ dans le théorème 1.1. Par contre, la formule donnant l'action de l'unipotent inférieur ne permet pas une déduction facile de la formule pour $u^{-}$.

Remarque 3.2. Les formules donnant l'action de $\mathrm{GL}_{2}\left(\mathbf{Q}_{p}\right)$ sont inspirées de celles obtenues en faisant agir $\mathrm{GL}_{2}\left(\mathbf{Q}_{p}\right)$ sur les mesures sur $\mathbf{P}^{1}\left(\mathbf{Q}_{p}\right)$. Rappelons que $L \otimes_{O_{L}} O_{L}[[T]]$ (resp. $\mathscr{R}^{+}=\mathscr{R} \cap L[[T]]$ ) s'identifie, grâce au théorème de Mahler (resp. Amice), à l'espace des mesures (resp. distributions) $\mu$ sur $\mathbf{Z}_{p}$, à valeurs dans $L$. L'identification se fait par la transformée d'Amice

$$
\mu \rightarrow \mathscr{A}_{\mu}=\sum_{n \geq 0} \int_{\mathbf{Z}_{p}}\left(\begin{array}{l}
x \\
n
\end{array}\right) \mu \cdot T^{n}=\int_{\mathbf{Z}_{p}}(1+T)^{x} \mu .
$$

Le monoïde $P^{+}$agit sur l'espace de ces mesures (resp. distributions) par

$$
\int_{\mathbf{Z}_{p}} \phi(x) g \cdot \mu=\int_{\mathbf{Z}_{p}} \phi(a x+b) \mu
$$

si $\phi: \mathbf{Z}_{p} \rightarrow L$ est continue (resp. localement analytique) et $g=\left(\begin{array}{ll}a & b \\ 0 & 1\end{array}\right)$. Au niveau des transformées d'Amice, l'action de $P^{+}$sur l'espace des mesures (resp. distributions) peut aussi s'écrire

$$
\mathscr{A}_{\left(\begin{array}{cc}
p^{k} & b \\
0 & 1
\end{array}\right) \mu}=(1+T)^{b} \cdot \varphi^{k}\left(\sigma_{a}\left(\mathscr{A}_{\mu}\right)\right)
$$

\footnotetext{
${ }^{14}$ La formule de [10 p. 325 comporte quelques erreurs de frappe.
} 
formule qui a un sens pour n'importe quel $(\varphi, \Gamma)$-module et qui a été utilisée dans l'introduction pour définir une action de $P^{+}$sur $\Delta$. Si $\mu$ est une mesure sur $\mathbf{Z}_{p}$ et $U$ est un ouvert compact de $\mathbf{Z}_{p}$, soit $\operatorname{Res}_{U}(\mu)$ la mesure telle que $\int_{\mathbf{Z}_{p}} \phi \operatorname{Res}_{U}(\mu)=\int_{\mathbf{Z}_{p}} 1_{U} \cdot \phi \mu$, où $1_{U}$ est la fonction caractèristique de $U$. Si $a \in \mathbf{Z}_{p}, n \geq 0$ et $\Lambda \in\left\{L \otimes O_{L} O_{L}[[T]], \mathscr{R}\right\}$, on définit $\operatorname{Res}_{a+p^{n}} \mathbf{Z}_{p}: \Lambda \rightarrow \Lambda$ par $\operatorname{Res}_{a+p^{n}} \mathbf{Z}_{p}=\left(\begin{array}{cc}1 & a \\ 0 & 1\end{array}\right) \circ \varphi^{n} \circ \psi^{n} \circ\left(\begin{array}{cc}1 & -a \\ 0 & 1\end{array}\right)$. Tout ouvert compact $U$ de $\mathbf{Z}_{p}$ peut s'écrire comme une réunion disjointe finie $U=\cup_{a \in I}\left(a+p^{n} \mathbf{Z}_{p}\right)$ et on pose $\operatorname{Res}_{U}=\sum_{a \in I} \operatorname{Res}_{a+p^{n} \mathbf{Z}_{p}}$, qui ne dépend pas du choix de la décomposition. On vérifie alors que $\operatorname{Res}_{U}\left(\mathscr{A}_{\mu}\right)=\mathscr{A}_{\operatorname{Res}_{U}(\mu)}$. Le faisceau équivariant attaché à $D$ est l'analogue du faisceau des mesures sur $\mathbf{Z}_{p}$ et de l'action de $P^{+}$que l'on vient de définir. L'espace $D \otimes \mathbf{P}^{1}$ est alors l'analogue de l'espace des mesures sur $\mathbf{P}^{1}\left(\mathbf{Q}_{p}\right)$, muni de l'action de $\mathrm{GL}_{2}\left(\mathbf{Q}_{p}\right)$ définie par

$$
\int_{\mathbf{P}^{1}\left(\mathbf{Q}_{p}\right)} \phi(x) g \cdot \mu=\int_{\mathbf{P}^{1}\left(\mathbf{Q}_{p}\right)} \delta_{D}(c x+d) \phi\left(\frac{a x+b}{c x+d}\right) \mu
$$

pour $g=\left(\begin{array}{ll}a & b \\ c & d\end{array}\right)$ et $\phi: \mathbf{Z}_{p} \rightarrow L$ continue. L'espace $\Delta \nabla \mathbf{Z}_{p}^{*}=\Delta^{\psi=0}$ correspond à l'espace des mesures à support dans $\mathbf{Z}_{p}^{*}$ et l'involution $w_{D}$ de $D \otimes \mathbf{Z}_{p}^{*}$ correspond au niveau des mesures à la transformée $\mu \rightarrow w_{D} \cdot \mu$ définie par

$$
\mathscr{A}_{w_{D} \cdot \mu}=\int_{\mathbf{Z}_{p}^{*}} \delta_{D}(x)(1+T)^{\frac{1}{x}} \mu .
$$

En approximant l'intégrale par des sommes de Riemann et developpement de Taylor à l'ordre 1 , on obtient une formule qui ne fait intervenir que l'action de $\varphi, \psi$ et $\Gamma$ sur $\mathscr{A}_{\mu}$. Cette formule a un sens pour tout $(\varphi, \Gamma)$-module $D$ et est donnée par

$$
w_{D}(z)=\lim _{n \rightarrow \infty} \sum_{i \in\left(\mathbf{Z} / p^{n} \mathbf{Z}\right)^{*}} \delta_{D}(i)(1+T)^{\frac{1}{i}} \sigma_{-\frac{1}{i^{2}}} \varphi^{n} \psi^{n}\left((1+T)^{-i} z\right)
$$

pour $z \in D^{\psi=0}$. Les résultats généraux de 9 , chap. $\mathrm{V}$ montrent que la limite existe et que $w_{D}$ est une involution continue de $D^{\psi=0}$. La série définissant $w_{D}(z)$ ne converge pas dans $D^{\dagger}$ ou $D_{\text {rijg. }}$ Cependant, si $D$ est attaché à une représentation de dimension 2, Colmez démontre15 (10], lemme V.2.4 et proposition V.2.9) que $w_{D}$ préserve $D^{\dagger, \psi=0}$ et se prolonge en une involution continue de $D_{\text {rig }}^{\psi=0}$. Cela permet de faire les mêmes constructions pour $D_{\text {rig. }}$.

\subsection{Actions infinitésimales sur le faisceau $U \rightarrow D_{\text {rig }} \otimes U$}

Si $m \geq 2$, soit $K_{m}=1+p^{m} M_{2}\left(\mathbf{Z}_{p}\right)$; c'est un groupe de Lie $p$-adique compact. Soit $\mathscr{D}\left(K_{m}\right)$ l'algèbre des distributions sur $K_{m}$ (voir [34], [35]), dual fort de l'algèbre $C^{\text {an }}\left(K_{m}, L\right)$ des fonctions localement analytiques sur $K_{m}$, à valeurs dans $L$. Soit $U\left(\mathfrak{g l}_{2}\right)$ l'algèbre enveloppante de l'algèbre de Lie de $\mathrm{GL}_{2}\left(\mathbf{Q}_{p}\right)$. On dispose d'une inclusion $U\left(\mathfrak{g l}_{2}\right) \subset \mathscr{D}\left(K_{m}, L\right)$, en voyant les éléments de $U\left(\mathfrak{g l}_{2}\right)$ comme des opérateurs différentiels sur $C^{\text {an }}\left(K_{m}, L\right)$ et en évaluant en $1 \in K_{m}$.

\footnotetext{
${ }^{15}$ Ce résultat est hautement nontrivial et découle essentiellement de la construction de la correspondance de Langlands locale $p$-adique, par prolongément analytique à partir du cas cristallin.
} 
Proposition 3.3. L'action de $\mathrm{GL}_{2}\left(\mathbf{Q}_{p}\right)$ sur $D_{\text {rig }} \otimes \mathbf{P}^{1}$ en fait un module sur $\mathscr{D}\left(K_{m}\right)$.

Démonstration. Passer à la limite inductive dans le lemme V.2.15 de [10] ou contempler le dernier isomorphisme de la remarque V.2.19 de [10]. Dans loc.cit., $m$ est supposé assez grand, mais comme les $K_{m}$ sont commensurables, cela ne change rien.

Lemme 3.4. Soit $H \subset \mathrm{GL}_{2}\left(\mathbf{Q}_{p}\right)$ un sous-groupe ouvert compact qui stabilise l'ouvert compact $U \subset \mathbf{P}^{1}\left(\mathbf{Q}_{p}\right)$. Alors $D_{\text {rig }} \otimes U \subset D_{\text {rig }} \otimes \mathbf{P}^{1}$ est stable par $\mathscr{D}(H)$ et on a $\operatorname{Res}_{U}(\lambda$. $z)=\lambda \cdot \operatorname{Res}_{U}(z)$ pour tout $z \in D_{\text {rig }} \otimes \mathbf{P}^{1}$ et tout $\lambda \in \mathscr{D}(H)$.

Démonstration. Si $\lambda \in L[H]$ est une combinaison linéaire de masses de Dirac, cela est évident par $\mathrm{GL}_{2}\left(\mathbf{Q}_{p}\right)$-équivariance de $\operatorname{Res}_{U}$ et le fait que $h(U)=U$ pour $h \in H$. Le résultat suit alors par continuité et densité de $L[H]$ dans $\mathscr{D}(H)$ ([34], lemme 3.1).

Le lemme précédent induit une action de $\mathfrak{g l}_{2}$ sur $D_{\text {rig }} \otimes \mathbf{Z}_{p}=D_{\text {rig }}$, qui satisfait $\operatorname{Res}_{U}(X \cdot z)=X \cdot \operatorname{Res}_{U}(z)$ pour $z \in D_{\text {rig }} \otimes \mathbf{P}^{1}, X \in \mathfrak{g l}_{2}$ et $U \subset \mathbf{Z}_{p}$ ouvert compact.

\subsection{L'action de l'élément de Casimir}

Rappelons que l'on utilise la base

$$
I_{2}=\left(\begin{array}{ll}
1 & 0 \\
0 & 1
\end{array}\right), \quad h=\left(\begin{array}{cc}
1 & 0 \\
0 & -1
\end{array}\right), \quad u^{+}=\left(\begin{array}{ll}
0 & 1 \\
0 & 0
\end{array}\right), \quad u^{-}=\left(\begin{array}{ll}
0 & 0 \\
1 & 0
\end{array}\right)
$$

de $\mathfrak{g l}_{2}$. Pour la définition des poids de Hodge-Tate généralisés d'une $L$-représentation $V$ et du polynôme $P_{\mathrm{Sen}, \mathrm{V}}$, voir la partie 2.5 .

Proposition 3.5. Si $k$ est la somme des poids de Hodge-Tate généralisés de $V$, alors pour tout $z \in D_{\text {rig }}$ on a $I_{2}(z)=(k-1) z, u^{+}(z)=t z$ et $h(z)=(1-k) z+2 \nabla z$.

Démonstration. La $\mathrm{GL}_{2}\left(\mathbf{Q}_{p}\right)$-représentation $D_{\text {rig }} \otimes \mathbf{P}^{1}$ a pour caractère central $\delta_{D}=$ $\chi^{-1} \cdot \operatorname{det} V$, qui, en tant que caractère galoisien, a poids de Hodge-Tate généralisé $k-1$ (car det $V$ a poids de Hodge-Tate $k$ ). Donc, en tant que caractère de $\mathbf{Q}_{p}^{*}$, la dérivée en 1 de $\delta_{D}$ est $k-1$. La formule $I_{2}(z)=(k-1) z$ s'en déduit. Comme $\left(\begin{array}{ll}1 & b \\ 0 & 1\end{array}\right) z=(1+T)^{b} z$ pour $z \in D_{\text {rig }}$ et $b \in p \mathbf{Z}_{p}$, on a

$$
u^{+}(z)=\lim _{b \rightarrow 0} \frac{(1+T)^{b}-1}{b} z=t z
$$

pour tout $z \in D_{\text {rig. }}$. Le caractère central de $D_{\text {rig }} \otimes \mathbf{P}^{1}$ étant $\delta_{D}$, un calcul immédiat donne

$$
\left(\begin{array}{ll}
a & 0 \\
0 & a^{-1}
\end{array}\right) z=\delta_{D}^{-1}(a) \sigma_{a^{2}}(z)
$$

Il s'ensuit que pour $z \in D_{\text {rig }}$ on a

$$
h(z)=\lim _{a \rightarrow 1} \frac{\delta_{D}^{-1}(a) \sigma_{a^{2}} z-z}{a-1}=(1-k) z+2 \nabla z,
$$

la dernière égalité étant une conséquence du fait que la dérivée de $\delta_{D}$ en 1 est $k-1$, comme on l'a expliqué ci-dessus. 
L'élément de Casimir

$$
C=u^{+} u^{-}+u^{-} u^{+}+\frac{1}{2} h^{2}
$$

engendre le centre de $U\left(\mathfrak{s l}_{2}\right)$. D'après le lemme 3.4 et la remarque qui le suit, on peut voir $C$ comme endomorphisme $L$-linéaire continu de $D_{\text {rig }}=D_{\text {rig }} \otimes \mathbf{Z}_{p}$.

Lemme 3.6. Soient a et $b$ les poids de Hodge-Tate généralisés de $V$, de telle sorte que $P_{\mathrm{Sen}, \mathrm{V}}(X)=(X-a)(X-b)$. On a une égalité d'opérateurs sur $D_{\mathrm{rig}}$

$$
C=2 t u^{-}+2 P_{\operatorname{Sen}, \mathrm{V}}(\nabla)+\frac{(a-b)^{2}-1}{2} .
$$

Démonstration. La proposition 3.5 montre qu'en tant qu'opérateurs sur $D_{\text {rig }}$ on a $h=$ $2 \nabla+1-k$ et $u^{+}(z)=t z$, où $k=a+b$. Un calcul immédiat montre que

$$
\frac{1}{2} h^{2}-h=\frac{(2 \nabla-k)^{2}-1}{2}=2 P_{\operatorname{Sen}, \mathrm{V}}(\nabla)+\frac{(a-b)^{2}-1}{2} .
$$

Le résultat suit alors de l'égalité $C=2 u^{+} u^{-}+\frac{1}{2} h^{2}-h$, déduite de $u^{+} u^{-}-u^{-} u^{+}=h$.

On est maintenant en mesure de démontrer le théorème principal de l'article. On garde les notations du lemme 3.6 .

Théorème 3.7. Pour tout $z \in D_{\text {rig }}$ on $a u^{-}(z)=-\frac{P_{\mathrm{Sen}, \mathrm{V}}(\nabla)(z)}{t}$ et $C(z)=\frac{(a-b)^{2}-1}{2} \cdot z$.

Démonstration. Comme $C$ commute à l'action adjointe de $\mathrm{GL}_{2}\left(\mathbf{Q}_{p}\right)$, les formules donnant l'action de $\mathrm{GL}_{2}\left(\mathbf{Q}_{p}\right)$ sur $D_{\text {rig }} \otimes \mathbf{P}^{1}$ montrent que l'opérateur $C: D_{\text {rig }} \rightarrow D_{\text {rig }}$ commute à $\varphi$ et $\Gamma$ et satisfait $C\left((1+T)^{m} \cdot z\right)=(1+T)^{m} \cdot C(z)$ pour tout $m \in \mathbf{N}$ et tout

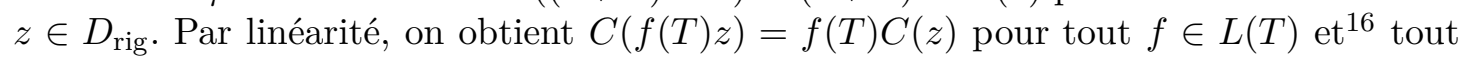
$z \in D_{\text {rig. }}$. Or, il est facile de voir que $L(T)$ est dense dans $\mathscr{R}$ (tout $f=\sum_{n \in \mathbf{Z}} a_{n} \cdot T^{n} \in \mathscr{R}$ est la limite dans $\mathscr{R}$ de la suite $\left.\sum_{|n| \leq N} a_{n} \cdot T^{n}\right)$. On en déduit que $C$ est $\mathscr{R}$-linéaire, i.e. $C \in \operatorname{End}_{\varphi, \Gamma, \mathscr{R}}\left(D_{\text {rig }}\right)=\operatorname{End}_{L\left[\mathrm{Gal}\left(\overline{\mathbf{Q}_{p}} / \mathbf{Q}_{p}\right)\right]}(V)$ (remarque 2.7). Comme $V$ est absolument irréductible, on conclut que $C$ est scalaire.

La proposition 2.14 combinée au lemme 3.6 montrent que $C-\frac{(a-b)^{2}-1}{2}$ envoie $D_{\text {rig }}$ dans $t \cdot D_{\text {rig. }}$ Comme de plus $C-\frac{(a-b)^{2}-1}{2}$ est scalaire, d'après la discussion précédente, on obtient finalement $C=\frac{(a-b)^{2}-1}{2}$. La formule pour $u^{-}$suit alors du lemme 3.6.

On finit par un corollaire concernant l'action de l'involution $w_{D}$ sur $D_{\text {rig }} \otimes \mathbf{Z}_{p}^{*}$.

Corollaire 3.8. Pour tout $z \in D_{\text {rig }} \otimes \mathbf{Z}_{p}^{*}$ on $a$

$$
w_{D}(t z)=-\frac{P_{\mathrm{Sen}, \mathrm{V}}(\nabla)\left(w_{D}(z)\right)}{t} .
$$

\footnotetext{
${ }^{16}$ Noter que si $z \in D_{\text {rig }}$ et $P \in L[T]$ est non nul, alors $\frac{1}{P(T)} \cdot z \in D_{\text {rig. }}$.
} 
Démonstration. Le théorème 3.7 montre que

$$
w_{D}(t z)=w_{D}\left(u^{+} z\right)=w u^{+} z=u^{-} w z=u^{-}\left(w_{D}(z)\right)=-\frac{P_{\operatorname{Sen}, \mathrm{V}}(\nabla)\left(w_{D}(z)\right)}{t} .
$$

\subsection{Application aux représentations de Banach}

Rappelons qu'une $L$-représentation de Banach de $\mathrm{GL}_{2}\left(\mathbf{Q}_{p}\right)$ est un $L$-espace de Banach $\Pi$ muni d'une action continue de $\mathrm{GL}_{2}\left(\mathbf{Q}_{p}\right)$. La représentation $\Pi$ est dite unitaire si elle admet une norme définissant la topologie de Banach et qui est invariante sous l'action de $\mathrm{GL}_{2}\left(\mathbf{Q}_{p}\right)$. Elle est dite admissible s'il existe une injection continue $H$-équivariante $\Pi \subset \mathscr{C}(H, L)^{n}$ pour un sous-groupe ouvert compact $H$ de $\mathrm{GL}_{2}\left(\mathbf{Q}_{p}\right)$ et un $n \geq 1$. Cela équivaut à ce que le dual topologique $\Pi^{*}$ de $\Pi$ soit un $L \otimes_{O_{I}} O_{L}\left[\left[\mathrm{GL}_{2}\left(\mathbf{Z}_{p}\right)\right]\right]$-module de type fini, où $O_{L}\left[\left[\mathrm{GL}_{2}\left(\mathbf{Z}_{p}\right)\right]\right]$ est l'algèbre de groupe complété 17 de $\mathrm{GL}_{2}\left(\mathbf{Z}_{p}\right)$.

Si $\Pi$ est une $\mathrm{GL}_{2}\left(\mathbf{Q}_{p}\right)$-représentation de Banach admissible, on note $\Pi^{\text {an }}$ le sousespace des vecteurs localement analytiques de $\Pi$ (ce sont les vecteurs $v \in \Pi$ tels que $g \rightarrow g \cdot v$ soit une fonction localement analytique sur $\mathrm{GL}_{2}\left(\mathbf{Q}_{p}\right)$, à valeurs dans le Banach $\Pi)$. D'après un théorème fondamental de Schneider et Teitelbaum ([35], theorem 7.1), il s'agit d'un sous-espace dense de $\Pi$. Le résultat suivant est dû à Colmez [10].

Théorème 3.9. Soit $V$ une L-représentation absolument irréductible, de dimension 2 de $\operatorname{Gal}\left(\overline{\mathbf{Q}_{p}} / \mathbf{Q}_{p}\right)$ et soient $D$ et $D_{\text {rig }}$ les $(\varphi, \Gamma)$-modules attachés à $V$. Il existe une $\mathrm{GL}_{2}\left(\mathbf{Q}_{p}\right)$ représentation de Banach unitaire admissible, topologiquement absolument irréductible $\Pi=\Pi(V)$ telle que l'on ait des suites exactes de $\mathrm{GL}_{2}\left(\mathbf{Q}_{p}\right)$-modules topologiques

$$
\begin{gathered}
0 \rightarrow \Pi^{*} \otimes\left(\delta_{D} \circ \text { det }\right) \rightarrow D \otimes \mathbf{P}^{1} \rightarrow \Pi \rightarrow 0, \\
0 \rightarrow\left(\Pi^{\text {an }}\right)^{*} \otimes\left(\delta_{D} \circ \text { det }\right) \rightarrow D_{\text {rig }} \otimes \mathbf{P}^{1} \rightarrow \Pi^{\text {an }} \rightarrow 0 .
\end{gathered}
$$

Remarque 3.10. Si $V, D$ et $\Pi$ sont comme dans le théorème 3.9 , on note $\check{\Pi}=\Pi\left(V^{*} \otimes \chi\right)$, où $V^{*}$ est le dual de $V$. Le choix d'un isomorphisme $\wedge^{2}(V) \simeq \chi \cdot \delta_{D}$ induit un isomorphisme $\check{\Pi} \simeq \Pi \otimes\left(\delta_{D}^{-1}\right.$ odet $)$, qui, combiné aux suites exactes du théorème 3.9, induit des inclusions $\mathrm{GL}_{2}\left(\mathbf{Q}_{p}\right)$-équivariantes $\check{\Pi}^{*} \subset D \otimes \mathbf{P}^{1}$ et $\left(\check{\Pi}^{\text {an }}\right)^{*} \subset D_{\text {rig }} \otimes \mathbf{P}^{1}$.

Le résultat suivant répond à une question de Harris ( [18], remark 3.3.8).

Théorème 3.11. Soit $V$ une L-représentation absolument irréductible de $G_{\mathbf{Q}_{p}}$, de dimension 2, à poids de Hodge-Tate généralisés a et b. Alors l'élément de Casimir agit par multiplication par $\frac{(a-b)^{2}-1}{2}$ sur $\Pi(V)^{\text {an }}$.

Démonstration. D'après le théorème [3.7, l'élément de Casimir agit par multiplication par $\frac{(a-b)^{2}-1}{2}$ sur $D_{\text {rig }} \otimes \mathbf{P}^{1}$. Comme $\Pi^{\text {an }}$ est un quotient de $D_{\text {rig }} \otimes \mathbf{P}^{1}$ d'après le théorème [3.9, le résultat suit.

\footnotetext{
${ }^{17}$ C'est la limite projective des algèbres de groupe des quotients finis de $\mathrm{GL}_{2}\left(\mathbf{Z}_{\mathrm{p}}\right)$. De manière plus conceptuelle, c'est l'algèbre des mesures à valeurs dans $O_{L}$ sur $\mathrm{GL}_{2}\left(\mathbf{Z}_{p}\right)$.
} 
Remarque 3.12. Dans [16], on montre que toute représentation localement analytique admissible et absolument irréductible d'un groupe de Lie $p$-adique admet un caractère infinitésima 18 et on conjecture que $\Pi^{\text {an }}$ admet un caractère infinitésimal pour toute représentation de Banach admissible et absolument irréductible $\Pi$. Le problème est que $\Pi^{\text {an }}$ n'est pas irréductible en général et les méthodes de [16] (qui utilisent pleinement les résultats de [1] et [35]) ne semblent pas s'adapter. Voici ce que l'on peut démontrer avec les résultats de cet article : si $p>3$ et si $\Pi$ est une $\mathrm{GL}_{2}\left(\mathbf{Q}_{p}\right)$-représentation de Banach unitaire, admissible, absolument irréductible, alors $\Pi^{\text {an }}$ admet un caractère infinitésimal. En effet, si $\Pi$ est un sous-quotient d'une induite unitaire parabolique, le résultat est facile et sinon, d'après un résultat de Paškūnas [30], conjecturé par Colmez, il existe une $L$-représentation $V$ absolument irréductible, de dimension 2 de $\operatorname{Gal}\left(\overline{\mathbf{Q}_{p}} / \mathbf{Q}_{p}\right)$ telle que $\Pi \simeq \Pi(V)$ et donc le résultat suit du théorème 3.11. Si la réduction modulo $p$ d'un réseau invariant de $\Pi$ est de longueur finie, on peut démontrer le même résultat sans l'hypothèse $p>3$ et sans utiliser le théorème de Paškūnas, mais la preuve reste bien détournée [17].

\section{Modèle de Kirillov et dualité}

Ce chapitre est préliminaire à la preuve du théorème 1.5. On commence par rappeler la construction d'un modèle de Kirillov pour les vecteurs de $\Pi$ qui sont algébriques sous l'action de l'unipotent supérieur. On rappelle ensuite le lien entre le modèle de Kirillov et la dualité entre $\Pi^{\text {an }}$ et $\left(\Pi^{\text {an }}\right)^{*}$, qui jouera un rôle crucial dans le chapitre suivant. Dans la suite on suppose que $V$ est absolument irréductible de dimension 2 , à poids de Hodge-Tate 0 et $k \in \mathbf{N}^{*}$ (en particulier, $V$ est de Hodge-Tate). Voir la définition 2.4 pour les $(\varphi, \Gamma)$-modules $D, D_{\text {rig }}, \tilde{D}, \tilde{D}^{+}, \tilde{D}_{\text {dif }}$ attachés à $V$. Soit $\Pi$ la représentation de Banach de $\mathrm{GL}_{2}\left(\mathbf{Q}_{p}\right)$ attachée à $V$ par la correspondance de Langlands locale $p$-adique (théorème 3.9). Soit $U$ le radical unipotent du Borel supérieur de $\mathrm{GL}_{2}\left(\mathbf{Q}_{p}\right)$ et soit $P$ le sous-groupe mirabolique de $\mathrm{GL}_{2}\left(\mathbf{Q}_{p}\right)$, formé des matrices du type $\left(\begin{array}{cc}a & b \\ 0 & 1\end{array}\right)$, avec $a \in \mathbf{Q}_{p}^{*}$ et $b \in \mathbf{Q}_{p}$. Pour les notations $\check{\Pi}$ et $\check{\Pi}^{\text {an }}$ voir la remarque 3.10 .

\subsection{Le module $N_{\text {dif }, n}$}

Le module $N_{\text {dif }, n}$ introduit dans cette partie va jouer un rôle crucial dans la suite. Il s'agit d'une version infinitésimale de l'équation différentielle attachée par Berger [2] à une représentation de de Rham.

Proposition 4.1. Posons $\varepsilon=1$ si $V$ n'est pas de Rham et 0 dans le cas contraire. Il existe $e_{1}, e_{2} \in D_{\mathrm{dif}, m(D)}^{+}[1 / t]$ tels que

a) $e_{1}, t^{k} e_{2}$ forment une base de $D_{\mathrm{dif}, n}^{+}$sur $L_{n}[[t]]$ pour tout $n \geq m(D)$.

b) $\sigma_{a}\left(e_{1}\right)=e_{1}$ et $\sigma_{a}\left(e_{2}\right)=e_{2}+\varepsilon \log a \cdot e_{1}$ pour tout $a \in 1+p^{m(D)} \mathbf{Z}_{p}$.

Démonstration. C'est le contenu de la proposition VI.3.2 de [10].

\footnotetext{
${ }^{18}$ On montre en fait que les endomorphismes d'une telle représentation sont tous scalaires.
} 
Définition 4.2. Les éléments $e_{1}, e_{2}$ de $D_{\mathrm{dif}, n}^{+}$étant comme dans la proposition 4.1, soit

$$
N_{\mathrm{dif}, n}=L_{n}[[t]] e_{1} \oplus L_{n}[[t]] e_{2} \subset t^{-k} D_{\mathrm{dif}, n}^{+} .
$$

Exemple 4.3. Si $V$ est de de Rham, on peut choisir pour $e_{2}$ une base de $D_{\mathrm{dR}}(V) / D_{\mathrm{dR}}^{+}(V)$, que l'on complète en une base $e_{1}, e_{2}$ de $D_{\mathrm{dR}}(V)$. On a alors $N_{\mathrm{dif}, n}=L_{n}[[t]] \otimes_{L} D_{\mathrm{dR}}(V)$ pour tout $n$ suffisament grand.

\subsection{Une caractérisation différentielle des représentations de de Rham}

Dans cette partie on donne un critère simple pour distinguer les représentations de de Rham parmi les représentations de Hodge-Tate. Ce genre d'argument est inspiré de [4], proposition 5.2.1. Notons $\nabla_{2 k}=\nabla(\nabla-1) \ldots(\nabla-2 k+1)$.

Lemme 4.4. Soit $n \geq m(D)$ et soient $e_{1}$ et $e_{2}$ comme dans la proposition 4.1. Notons

$$
X_{n}=\left\{z \in D_{\mathrm{dif}, n}^{+} \mid \nabla_{2 k}(z) \in t^{2 k} N_{\mathrm{dif}, n}\right\},
$$

a) Si $V$ est de de Rham, alors $X_{n}=D_{\mathrm{dif}, n}^{+}$.

b) Si $V$ est de Hodge-Tate mais pas de de Rham, alors $X_{n}=L_{n}[[t]] e_{1}+t^{2 k} L_{n}[[t]] e_{2}$.

Démonstration. Notons que $\nabla_{2 k}\left(t^{j}\right)=j(j-1) \ldots(j-2 k+1) t^{j} \in t^{2 k} L_{n}[[t]]$ pour tout $j \geq 0$. La partie a) suit alors de cette observation et du fait que pour tous $A, B \in L_{n}[[t]]$ on a (noter que $\left.\nabla e_{1}=\nabla e_{2}=0\right)$

$$
\nabla_{2 k}\left(A(t) e_{1}+B(t) e_{2}\right)=\nabla_{2 k}(A(t)) e_{1}+\nabla_{2 k}(B(t)) e_{2} .
$$

Supposons donc que $V$ n'est pas de de Rham, ce qui implique les égalités $\nabla\left(e_{2}\right)=e_{1}$ et $\nabla\left(e_{1}\right)=0$. D'après la règle de Leibnitz, on a pour tout $B \in L_{n}[[t]]$ et tout $j \geq 0$

$$
\nabla^{j}\left(B e_{2}\right)=\nabla^{j}(B) e_{2}+j \nabla^{j-1}(B) e_{1} .
$$

On en déduit que pour tout $P \in L[[T]]$ on a $P(\nabla)\left(B e_{2}\right)=(P(\nabla) B) e_{2}+\left(P^{\prime}(\nabla) B\right) e_{1}$, donc

$$
\nabla_{2 k}\left(B e_{2}\right)=\left(\nabla_{2 k} B\right) e_{2}+\left(\sum_{j=0}^{2 k-1} \frac{\nabla_{2 k}}{\nabla-j} B\right) e_{1} .
$$

Comme $\nabla_{2 k}\left(L_{n}[[t]]\right) \subset t^{2 k} L_{n}[[t]]$, on obtient que $x=A e_{1}+B e_{2}$ est dans $X_{n}$ si et seulement si

$$
\sum_{j=0}^{2 k-1} \frac{\nabla_{2 k}}{\nabla-j} B \in t^{2 k} L_{n}[[t]] .
$$

Mais si $B=\sum_{j \geq 0} \alpha_{j} t^{j}$, alors on a des égalités dans $L_{n}[[t]] / t^{2 k} L_{n}[[t]]$

$$
\sum_{j=0}^{2 k-1} \frac{\nabla_{2 k}}{\nabla-j} B=\sum_{s=0}^{2 k-1} \alpha_{s} \cdot \sum_{j=0}^{2 k-1} \frac{\nabla_{2 k}}{\nabla-j}\left(t^{s}\right)
$$




$$
=\sum_{s=0}^{2 k-1} \alpha_{s} \cdot \frac{\nabla_{2 k}}{\nabla-s}\left(t^{s}\right)=\sum_{s=0}^{2 k-1}(-1)^{2 k-1-s} s ! \cdot(2 k-s-1) ! \alpha_{s} \cdot t^{s} .
$$

On a donc $x \in X_{n}$ si et seulement si $\alpha_{s}=0$ pour tout $0 \leq s \leq 2 k-1$, i.e. si et seulement si $B \in t^{2 k} L_{n}[[t]]$. Cela permet de conclure.

\subsection{Les accouplements $[,]_{\mathbf{P}^{1}}$ et $[,]_{\text {dif }}$}

Soit $V$ comme dans l'introduction de ce chapitre et identifions $\wedge^{2}(V)$ à $L$ par le choix d'une base de $\wedge^{2}(V)$. L'accouplement $V \times V \rightarrow L$ donné par $(x, y) \rightarrow x \wedge y$ induit, par fonctorialité, un accouplement $D \times D \rightarrow \mathscr{E}$, noté encore $(x, y) \rightarrow x \wedge y$, qui satisfait 19 (pour $x, y \in D$ et $a \in \mathbf{Z}_{p}^{*}$ )

$$
\sigma_{a}(x) \wedge \sigma_{a}(y)=a \delta_{D}(a) \cdot \sigma_{a}(x \wedge y), \quad \varphi(x) \wedge \varphi(y)=\delta_{D}(p) \cdot \varphi(x \wedge y) .
$$

Notons $\operatorname{res}_{0}\left(f \frac{d T}{1+T}\right)$ le résidu en 0 de la forme différentielle $\frac{f}{1+T} d T$. On définit un accouplement [, ]:D $\times D \rightarrow L$ par la formule

$$
[x, y]=\operatorname{res}_{0}\left(\left(\sigma_{-1}(x) \wedge y\right) \frac{d T}{1+T}\right),
$$

ainsi qu'un accouplement $\left[,{ }_{\mathbf{P}^{1}}:\left(D \otimes \mathbf{P}^{1}\right) \times\left(D \otimes \mathbf{P}^{1}\right) \rightarrow L\right.$ par la formule

$$
\left[\left(x_{1}, x_{2}\right),\left(y_{1}, y_{2}\right)\right]_{\mathbf{P}^{1}}=\left[x_{1}, y_{1}\right]+\left[\varphi \psi\left(x_{2}\right), \varphi \psi\left(y_{2}\right)\right] .
$$

Sa restriction à $D \times D$ est l'accouplement [, ]. Cette discussion s'applique aussi à $D_{\text {rig }}$.

Proposition 4.5. 1) [ , $]_{\mathbf{P}^{1}}$ est un accouplement parfait de L-espaces vectoriels topologiques, satisfaisant $[g x, g y]_{\mathbf{P}^{1}}=\delta_{D}(\operatorname{det} g)[x, y]_{\mathbf{P}^{1}}$ pour tous $x, y \in \Delta$ et $g \in \mathrm{GL}_{2}\left(\mathbf{Q}_{p}\right)$.

2) $\check{\Pi}^{*}$ (resp. $\left.\left(\check{\Pi}^{\mathrm{an}}\right)^{*}\right)$ est son propre orthogonal dans $D \otimes \mathbf{P}^{1}\left(\right.$ resp. $\left.D_{\text {rig }} \otimes \mathbf{P}^{1}\right)$.

Démonstration. Toutes les références sont à [10] (on a $[x, y]=\left\{x \otimes \delta_{D}^{-1}, y\right\}$ avec les notations de loc.cit). 1) est la réunion des propositions I.2.1, I.2.2, II.1.11 (et des remarques faites avant la proposition V.2.10), et du théorème II.1.13. Ensuite, 2) est la réunion du théorème II.2.11, de la proposition V.2.10 et de la remarque V.2.21.

On déduit du 1) de la proposition précèdente que pour tout $k \geq 1$ et $x, y \in D_{\text {rig }}$ on a

$$
\left[\left(u^{-}\right)^{k} x, y\right]_{\mathbf{P}^{1}}=(-1)^{k}\left[x,\left(u^{-}\right)^{k} y\right]_{\mathbf{P}^{1}} .
$$

L'accouplement $V \times V \rightarrow L$ induit aussi un accouplement $\tilde{D}_{\text {dif }} \times \tilde{D}_{\text {dif }} \rightarrow L \otimes \mathbf{Q}_{p} \mathbf{B}_{\mathrm{dR}}^{H}$, noté $(x, y) \rightarrow x \wedge y$, satisfaisant $\sigma_{a}(x) \wedge \sigma_{a}(y)=a \delta_{D}(a) \cdot \sigma_{a}(x \wedge y)$ pour $x, y \in \tilde{D}_{\text {dif }}$ et $a \in \mathbf{Z}_{p}^{*}$. Soit $\operatorname{Res}_{\mathbf{Z}_{p}}: L \otimes \mathbf{Q}_{p} \mathbf{B}_{\mathrm{dR}}^{H} \rightarrow L((t))$ la trace de Tate normalisée (voir [2.6) et,

\footnotetext{
${ }^{19}$ Cette torsion par $\chi \cdot \delta_{D}$ vient du fait que l'accouplement $V \times V \rightarrow L$ n'est pas Galois-équivariant, mais il le devient si l'on tord par $\chi \cdot \delta_{D}$.
} 
si $f=\sum_{n>>-\infty} a_{n} t^{n} \in L((t))$, notons $\operatorname{res}_{0}(f d t)=a_{-1}$. On définit un accouplement $[,]_{\text {dif }}: \tilde{D}_{\text {dif }} \times \tilde{D}_{\text {dif }} \rightarrow L$ par

$$
[x, y]_{\operatorname{dif}}=\operatorname{res}_{0}\left(\operatorname{Res}_{\mathbf{Z}_{p}}\left(\sigma_{-1}(x) \wedge y\right) d t\right) .
$$

Le résultat suivant se déduit20 de [10, lemme VI.4.16].

Lemme 4.6. L'orthogonal dans $D_{\mathrm{dif}, n}^{+}[1 / t]$ de $N_{\mathrm{dif}, n}$ est $t^{k} N_{\mathrm{dif}, n}$ et $D_{\mathrm{dif}, n}^{+}$est son propre orthogonal dans $D_{\mathrm{dif}, n}^{+}[1 / t]$.

La trace de Tate $\frac{1}{p} \operatorname{Tr}_{L_{n+1} / L_{n}}$ envoie $D_{\text {dif,n+1 }}^{+} / t^{k} N_{\text {dif,n+1 }}$ dans $D_{\text {dif }, n}^{+} / t^{k} N_{\text {dif }, n}$. Si $x=$ $\left(x_{n}\right)_{n} \in \lim _{\longleftarrow} D_{\text {dif }, n}^{+} / t^{k} N_{\text {dif }, n}$ (la limite est relativement aux traces de Tate) et si $y \in$ $X_{\infty}^{-}=\underset{\lim }{\longrightarrow} N_{\text {dif }, n} / D_{\text {dif }, n}^{+}$, on définit $[x, y]_{\text {dif }}$ de la façon suivante : on choisit $n$ tel que $y \in N_{\mathrm{dif}, n} / D_{\mathrm{dif}, n}^{+}$, ainsi que des relèvements $\hat{y}_{n} \in N_{\mathrm{dif}, n}$ de $y$ et $\hat{x}_{n} \in D_{\mathrm{dif}, n}^{+}$de $x_{n}$ et on pose $[x, y]_{\text {dif }}=\left[\hat{x}_{n}, \hat{y}_{n}\right]_{\text {dif }}$.

Lemme 4.7. Cet accouplement est bien défini et non dégénéré.

Démonstration. Le fait que $[x, y]_{\text {dif }}$ ne dépend pas des choix de $\hat{x}_{n}$ ou $\hat{y}_{n}$ découle du lemme 4.6. Pour montrer qu'il ne dépend pas de $n$, il suffit de voir que si $\hat{y}_{n+1}-\hat{y}_{n} \in$ $D_{\text {dif }, \mathrm{n}+1}^{+}$et $\hat{x}_{n+1}, \hat{x}_{n}$ s'envoient sur $x_{n+1}, x_{n}$, alors $\left[\hat{x}_{n+1}, \hat{y}_{n+1}\right]_{\text {dif }}=\left[\hat{x}_{n}, \hat{y}_{n}\right]_{\text {dif }}$. Or, $\hat{y}_{n+1}-$ $\hat{y}_{n}$ étant dans $D_{\text {dif,n+1 }}^{+}$, il est orthogonal à $\hat{x}_{n+1}$, donc il reste à voir que $\hat{y}_{n}$ est orthogonal à $\hat{x}_{n+1}-\hat{x}_{n}$. Comme $p^{-1} \operatorname{Tr}_{L_{n+1} / L_{n}}\left(x_{n+1}\right)=x_{n}$, il reste en fait à voir que si $y \in N_{\text {dif }, n}$ et $x \in D_{\text {dif, } \mathrm{n}+1}^{+}$, alors $p^{-1} \operatorname{Tr}_{L_{n+1} / L_{n}}(x)-x$ est orthogonal à $y$. Cela est immédiat sur la formule définissant l'accouplement. Le fait que l'accouplement est non dégénéré suit du lemme 4.6 .

\subsection{La restriction de $\Pi$ au mirabolique}

Rappelons que $H=\operatorname{Gal}\left(\overline{\mathbf{Q}_{p}} / \mathbf{Q}_{p}\left(\mu_{p^{\infty}}\right)\right)=\operatorname{Ker} \chi$. Si $b \in \mathbf{Q}_{p}$, soit $n$ tel que $p^{n} b \in \mathbf{Z}_{p}$ et posons

$$
\left[(1+T)^{b}\right]=\varphi^{-n}\left((1+T)^{p^{n} b}\right)=\varphi^{-n}\left(\sum_{k \geq 0}\left(\begin{array}{c}
p^{n} b \\
k
\end{array}\right) T^{k}\right) .
$$

On obtient ainsi un élément de $\left(\tilde{\mathbf{B}}^{+}\right)^{H}$, qui ne dépend pas du choix de $n$. L'application $b \rightarrow\left[(1+T)^{b}\right]$ est un analogue $p$-adique de $x \rightarrow e^{2 i \pi x}$ et la transformée de Fourier $\mu \rightarrow \int_{\mathbf{Q}_{p}}\left[(1+T)^{x}\right] \mu$ identifie $L \otimes_{\mathbf{Q}_{p}}\left(\tilde{\mathbf{B}}^{+}\right)^{H}$ à l'espace des mesures sur $\mathbf{Q}_{p}$, nulles à l'infini (voir la proposition IV.3.1 de [9]). Comme $\varphi$ est inversible sur $\tilde{D}^{+}=\left(\tilde{\mathbf{B}}^{+} \otimes_{\mathbf{Q}_{p}} V\right)^{H}$ et $\tilde{D}=\left(\tilde{\mathbf{B}} \otimes \mathbf{Q}_{p} V\right)^{H}$, on peut munir ces deux modules d'une action du mirabolique $P$ grâce à $\left(\right.$ si $\left.k \in \mathbf{Z}, a \in \mathbf{Z}_{p}^{*}, b \in \mathbf{Q}_{p}\right)$

$$
\left(\begin{array}{cc}
p^{k} a & b \\
0 & 1
\end{array}\right) \tilde{z}=\left[(1+T)^{b}\right] \varphi^{k}\left(\sigma_{a}(\tilde{z})\right) .
$$

\footnotetext{
${ }^{20}$ C'est une simple traduction.
} 
D'après [9, lemme IV.1.2], tout élément $z$ de $\tilde{D}$ s'écrit de manière unique sous la forme $z=\sum_{i \in I}\left[(1+T)^{i}\right] z_{i}$, où $I \subset \mathbf{Q}_{p}$ est un système de représentants de $\mathbf{Q}_{p} / \mathbf{Z}_{p}$ et $z_{i} \in D$ tend vers 0 quand $i \rightarrow \infty$ dans $\mathbf{Q}_{p}$. Soit $I_{n}=I \cap p^{-n} \mathbf{Z}_{p}$ et notons

$$
x_{n}(z)=\sum_{i \in I_{n}}\left(\begin{array}{ll}
1 & i \\
0 & 1
\end{array}\right) z_{i} \in D \otimes p^{-n} \mathbf{Z}_{p} \subset D \otimes \mathbf{P}^{1} .
$$

D'après le lemme II.1.16 de [10], la suite $\left(x_{n}(z)\right)_{n>0}$ d'éléments de $D \otimes \mathbf{P}^{1}$ converge vers un élément que l'on note encore $z \in D \otimes \mathbf{P}^{1}$ et l'application $\tilde{D} \rightarrow D \otimes \mathbf{P}^{1}$ ainsi obtenue est une injection $P$-équivariante. Le corollaire II.2.8 de [10] montre que cette inclusion induit une inclusion $\tilde{D}^{+} \subset \check{\Pi}^{*}$ (en fait, le lemme cité montre que l'on a même $\left.\check{\Pi}^{*}=\tilde{D}^{+}+w \cdot \tilde{D}^{+}\right)$. Le résultat suivant ([10], cor. II.2.9) sera très utile pour la suite.

Proposition 4.8. L'inclusion de $\tilde{D}$ dans $D \otimes \mathbf{P}^{1}$ induit un isomorphisme de P-modules de Banach $\tilde{D} / \tilde{D}^{+} \simeq \Pi$.

\subsection{Le modèle de Kirillov de Colmez}

Définition 4.9. a) $v \in \Pi$ est dit $U$-algébrique s'il existe $n$ tel que $\left(\left(\begin{array}{ll}1 & p^{n} \\ 0 & 1\end{array}\right)-1\right)^{k} v=0$, ce qui équivaut à ce que $x \rightarrow\left(\begin{array}{ll}1 & x \\ 0 & 1\end{array}\right) v$ soit localement polynomiale de degré plus petit que $k$. On note $\Pi^{U \text {-alg }}$ l'espace des vecteurs $U$-algébriques.

b) $v \in \Pi$ est dit $P$-algébrique si $v$ est $U$-algébrique et s'il existe $n$ tel que $\lambda_{n}(v)=0$, où

$$
\lambda_{n}=\prod_{i=0}^{k-1}\left(\left(\begin{array}{rr}
1+p^{n} & 0 \\
0 & 1
\end{array}\right)-\left(1+p^{n}\right)^{i}\right) \in O_{L}\left[\mathrm{GL}_{2}\left(\mathbf{Z}_{p}\right)\right] .
$$

Cela équivaut à ce que les applications $x \rightarrow\left(\begin{array}{cc}1 & x \\ 0 & 1\end{array}\right) v$ et $x \rightarrow\left(\begin{array}{cc}x & 0 \\ 0 & 1\end{array}\right) v$ soient localement polynomiales de degré plus petit que $k$. On note $\Pi^{P-\text { alg }}$ l'espace des vecteurs $P$-algébriques.

Soit $v \in \Pi$ et soit21 $\tilde{z} \in \tilde{D}$ un relèvement de $v$. Comme $\left(\begin{array}{ll}1 & b \\ 0 & 1\end{array}\right)$ agit sur $\tilde{D}$ par multiplication par $(1+T)^{b}$ pour $b \in \mathbf{Z}_{p}$, on a $v \in \Pi^{U-\text { alg }}$ si et seulement si $\tilde{z} \in \frac{1}{\varphi^{n}(T)^{k}} \cdot \tilde{D}^{+}$pour un certain $n$. Puisque $\varphi^{n}(T)$ divise $t$ dans $\mathbf{B}_{\mathrm{dR}}^{+}$et $\tilde{\mathbf{B}}^{+} \subset \mathbf{B}_{\mathrm{dR}}^{+}$, on obtient $\tilde{z} \in t^{-k} \tilde{D}_{\mathrm{dif}}^{+}$.

Comme $\left(\begin{array}{ll}x & 0 \\ 0 & 1\end{array}\right) \tilde{z}\left(\bmod \tilde{D}^{+}\right)$reste $U$-algébrique pour tout $x \in \mathbf{Q}_{p}^{*}$, cela nous fournit une application

$$
\phi_{v}: \mathbf{Q}_{p}^{*} \rightarrow t^{-k} \tilde{D}_{\text {dif }}^{+} / \tilde{D}_{\text {dif }}^{+}, \quad \phi_{v}(x)=\left(\begin{array}{cc}
x & 0 \\
0 & 1
\end{array}\right) \tilde{z} \quad\left(\bmod \tilde{D}_{\text {dif }}^{+}\right) .
$$

Le résultat suivant en résume les propriétés essentielles.

Proposition 4.10. Soit $v \in \Pi^{U-\text { alg }}$.

1) $\phi_{v}$ est bien définie (i.e. ne dépend pas du choix de $\tilde{z}$ ), à support compact dans $\mathbf{Q}_{p}$ et $v \rightarrow \phi_{v}$ est injective.

2) On $a \sigma_{a}\left(\phi_{v}(x)\right)=\phi_{v}(a x)$ pour $a \in \mathbf{Z}_{p}^{*}$ et $x \in \mathbf{Q}_{p}^{*}$. De plus, si $g=\left(\begin{array}{ll}a & b \\ 0 & d\end{array}\right)$, on $a$

$$
\phi_{g v}(x)=\delta_{D}(d)\left[(1+T)^{\frac{b x}{d}}\right] \phi_{v}\left(\frac{a x}{d}\right) .
$$

\footnotetext{
${ }^{21}$ Rappelons que d'après la proposition 4.8 la représentation $\Pi$ restreinte à $P$ est isomorphe à $\tilde{D} / \tilde{D}^{+}$.
} 
3) $\Pi^{P-a l g}$ est stable par le Borel supérieur et

$$
\Pi^{P-\text { alg }}=\left\{v \in \Pi^{U-\text { alg }} \mid \quad \operatorname{Im}\left(\phi_{v}\right) \subset X_{\infty}^{-}\right\},
$$

où $X_{\infty}^{-}$est la réunion croissante des sous-espaces $N_{\mathrm{dif}, n} / D_{\mathrm{dif}, n}^{+}$de $t^{-k} \tilde{D}_{\mathrm{dif}}^{+} / \tilde{D}_{\mathrm{dif}}^{+}$.

Démonstration. Voir les lemmes VI.5.4 et VI.5.5 de [10].

Définition 4.11. Soit $\Pi_{c}^{P-a l g}$ l'espace des vecteurs $v \in \Pi^{P-\text { alg }}$ tels que $\phi_{v}$ soit à support compact dans $\mathbf{Q}_{p}^{*}$ (i.e. $\phi_{v}$ est nulle sur $p^{n} \mathbf{Z}_{p}-\{0\}$ pour tout $n$ assez grand).

\subsection{Dualité et modèle de Kirillov}

Le corollaire II.7.2 et le lemme V.2.15 de [10] montrent que $\operatorname{Res}_{\mathbf{Z}_{p}}\left(\left(\check{\Pi}^{\text {an }}\right)^{*}\right) \subset D^{\left.] 0, r_{m(D)}\right]}$. On peut donc définir pour $n \geq m(D), l \in \mathbf{Z}$ et $z \in\left(\check{\Pi}^{\text {an }}\right)^{*}$ la quantité

$$
i_{l, n}(z)=\varphi^{-n}\left(\operatorname{Res}_{\mathbf{Z}_{p}}\left(\left(\begin{array}{cc}
p^{n-l} & 0 \\
0 & 1
\end{array}\right) z\right)\right) \in D_{\mathrm{dif}, n}^{+} .
$$

Pour tout $z \in D^{\left.0, r_{n+1}\right]}$ et $n \geq m(D)$, on a

$$
\varphi^{-n}(\psi(z))=p^{-1} \operatorname{Tr}_{L_{n+1} / L_{n}} \varphi^{-(n+1)}(z) .
$$

De plus, pour tout $x \in D_{\text {rig }} \otimes \mathbf{P}^{1}$ on a

$$
\operatorname{Res}_{\mathbf{Z}_{p}}\left(\left(\begin{array}{cc}
p^{-1} & 0 \\
0 & 1
\end{array}\right) x\right)=\psi\left(\operatorname{Res}_{\mathbf{Z}_{p}}(x)\right)
$$

On en déduit que $\frac{1}{p} \operatorname{Tr}_{L_{n+1} / L_{n}}\left(i_{l, n+1}(z)\right)=i_{l, n}(z)$ pour $n \geq m(D), l \in \mathbf{Z}$ et $z \in\left(\check{\Pi}^{\text {an }}\right)^{*}$. On dispose donc d'un élément22

$$
i_{l}^{+}(z)=\left(i_{l, n}(z) \quad\left(\bmod t^{k} N_{\mathrm{dif}, n}\right)\right)_{n \geq m(D)} \in \lim _{\longleftarrow} D_{\mathrm{dif}, n}^{+} / t^{k} N_{\mathrm{dif}, n}
$$

Cette observation et le 3) de la proposition 4.10 donnent un sens à l'énoncé suivant (pour la définition des accouplements $[,]_{\mathbf{P}^{1}}$ et $[,]_{\text {dif }}$ voir 4.3$)$.

Proposition 4.12. $\Pi_{c}^{P-a l g}$ est contenu dans $\Pi^{\text {an }}$ et pour tout $z \in\left(\check{\Pi}^{\text {an }}\right)^{*}$ et $v \in \Pi_{c}^{P-a l g}$ on $a$

$$
[z, v]_{\mathbf{P}^{1}}=\sum_{l \in \mathbf{Z}} \delta_{D}\left(p^{l}\right)\left[i_{l}^{+}(z), \phi_{v}\left(p^{-l}\right)\right]_{\mathrm{dif}} .
$$

Démonstration. C'est le contenu de la proposition VI.5.12 de [10], dont l'ingrédient de base est la section VI.1 de [10] (elle est donc indépendante du reste du chapitre VI).

\footnotetext{
${ }^{22}$ Les applications de transition dans $\lim D_{\mathrm{dif}, n}^{+} / t^{k} N_{\mathrm{dif}, n}$ sont les traces de Tate normalisées.
} 


\section{Vecteurs localement algébriques et représentations de de Rham}

Le but de ce chapitre est de démontrer le théorème 1.5. L'ingrédient principal est l'étude de $\Pi_{c}^{P \text {-alg }}$, étude qui se fait d'une part grâce à la théorie de Hodge $p$-adique (proposition 5.4), de l'autre en reliant le modèle de Kirillov et la dualité entre $\Pi^{\text {an }}$ et $\left(\Pi^{\text {an }}\right)^{*}$ (proposition 4.12). Une fois ces résultats établis, le théorème 1.5] est une conséquence plus ou moins formelle du théorème 1.1. Dans ce chapitre $V$ est une $L$-représentation absolument

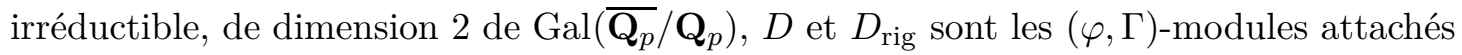
à $V$ et $\Pi$ est la $\mathrm{GL}_{2}\left(\mathbf{Q}_{p}\right)$-représentation de Banach attachée à $V$ (théorème 3.9).

\subsection{Sorites sur les vecteurs localement algébriques}

Soit $G$ un $\mathbf{Q}_{p}$-groupe algébrique réductif, que l'on identifie à ses $\mathbf{Q}_{p}$-points et soit $\pi$ une $L$-représentation localement analytique de $G$ (au sens de [34]). Soit $\mathfrak{g}=\operatorname{Lie}(G)$ et $\operatorname{Rep}_{L}^{\mathrm{alg}}(G)$ la catégorie des $L$-représentations du groupe algébrique $G$.

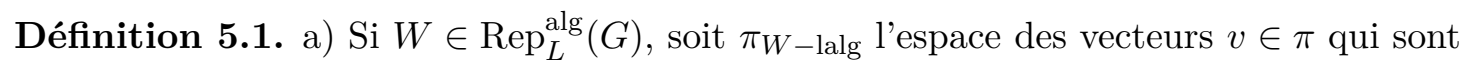
dans l'image d'un morphisme $H$-équivariant $f: W^{n} \rightarrow \pi$, pour un sous-groupe ouvert $H$ de $G$ et un entier $n \geq 1$. C'est un sous-espace de $\pi$ stable par $G$.

b) On dit que $v \in \pi$ est localement algébrique et on écrit $v \in \pi^{\text {alg }}$ s'il existe $W \in$

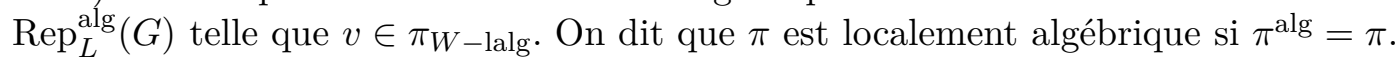

Cette définition, tirée de [20] est équivalente aux celles utilisées par SchneiderTeitelbaum [33] ou Colmez [10], grâce à la proposition 4.2.8 de [20]. Soit $\operatorname{Hom}(W, \pi)^{\mathfrak{g}}$ l'espace des morphismes $\mathfrak{g}$-équivariants de $W$ dans $\pi$.

Proposition 5.2. a) Soit $W \in \operatorname{Rep}_{L}^{\operatorname{alg}}(G)$. Le morphisme naturel

$$
\operatorname{Hom}(W, \pi)^{\mathfrak{g}} \otimes_{L} W \rightarrow \pi_{W-\mathrm{lalg}}
$$

est un isomorphisme topologique et $\pi_{W-l a l g}$ est un sous-espace fermé de $\pi$.

b) Soit $\hat{G}$ un système de représentants des objets irréductibles de $\operatorname{Rep}_{L}^{\text {alg }}(G)$. L'application naturelle

est un isomorphisme.

$$
\oplus_{W \in \hat{G}} \pi_{W-\mathrm{lalg}} \rightarrow \pi^{\mathrm{alg}}
$$

Démonstration. Voir la section 4.2 de [20].

Le résultat suivant est dû à Colmez [10, prop. VI.5.1]. Nous en donnons une démonstration plus directe, basée sur le théorème 3.11. On note $\operatorname{Sym}^{k-1}\left(L^{2}\right)$ la puissance symétrique $(k-1)$-ième de la représentation standard de $\mathrm{GL}_{2}\left(\mathbf{Q}_{\mathrm{p}}\right)$ sur $L \oplus L$.

Proposition 5.3. a) Si $\Pi^{\mathrm{alg}} \neq 0$, alors $V$ est Hodge-Tate à poids distincts.

b) Si les poids de Hodge-Tate de $V$ sont 0 et $k \in \mathbf{N}^{*}$ et si $\Pi(V)^{\text {alg }} \neq 0$, alors

$$
\Pi^{\mathrm{alg}}=\operatorname{Sym}^{k-1}\left(L^{2}\right) \otimes_{L} \Pi^{\mathrm{lc}}
$$

pour une représentation lisse admissible $\Pi^{\mathrm{lc}}$. 
Démonstration. Si $k \geq 1$ et $l$ sont des entiers, soit $W_{l, k}=\operatorname{Sym}^{k-1} L^{2} \otimes \operatorname{det}^{l}$. Supposons que $W_{l, k} \otimes \Pi^{\text {lc }}$ est une sous-représentation de $\Pi$, pour une représentation lisse $\Pi^{\text {lc }}$. Soient $a$ et $b$ les poids de Hodge-Tate de $V$. En considérant les caractères centraux, on obtient $a+b=k+2 l$. L'examen des caractères infinitésimaux (en utilisant le théorème 3.11) fournit $k^{2}=(a-b)^{2}$. Comme $k$ est un entier strictement positif, cela montre que $a$ et $b$ sont des entiers distincts et que $k$ et $l$ sont uniquement détérminés par $a$ et $b$. Ceci et la proposition 5.2 permettent de conclure (l'admissibilité de $\Pi^{\text {lc }}$ suit de celle de $\Pi$, car $\Pi^{\mathrm{lc}}=\operatorname{Hom}\left(\operatorname{Sym}^{k-1}\left(L^{2}\right), \Pi^{\mathrm{an}}\right)^{\mathfrak{g}}$ est un sous-espace fermé de $\operatorname{Hom}\left(\operatorname{Sym}^{k-1}\left(L^{2}\right), \Pi^{\mathrm{an}}\right)$, qui est une représentation localement analytique admissible puisque $\operatorname{Sym}^{k-1}\left(L^{2}\right)$ est de dimension finie; on conclut alors par la proposition 6.4 et le théorème 6.6 de [35]).

\subsection{L'espace $\Pi_{c}^{P-\text { alg }}$ et vecteurs presque algébriques}

La proposition suivante est due à Colmez [10, lemme VI.5.10]. On en donne une autre démonstration. L'espace $\Pi_{c}^{P-\text { alg }}$ a été défini dans 4.11 .

Proposition 5.4. L'application $v \rightarrow\left(\phi_{v}\left(p^{i}\right)\right)_{i \in \mathbf{Z}}$ induit une bijection de $\Pi_{c}^{P-\text { alg }}$ sur $\oplus_{i \in \mathbf{Z}} X_{\infty}^{-}$(voir la proposition 4.10, 3) pour $X_{\infty}^{-}$).

Démonstration. L'image est bien contenue dans $\oplus_{i \in \mathbf{Z}} X_{\infty}^{-}$, car $\operatorname{Im}\left(\phi_{v}\right) \subset X_{\infty}^{-}$(prop 4.10) et $\phi_{v}\left(p^{i}\right)=0$ pour $|i|>>$ (par définition). Si $\phi_{v}\left(p^{i}\right)=0$ pour tout $i$, on a aussi $\phi_{v}(x)=0$ pour tout $x$ d'après le 2) de la prop. 4.10 et l'injectivité de $v \rightarrow \phi_{v}$ montre que $v=0$. La partie délicate est la surjectivité. Pour cela, notons d'abord qu'il suffit de montrer que pour tout $x \in X_{\infty}^{-}$, on peut trouver $v \in \Pi_{c}^{P-a l g}$ tel que $\phi_{v}\left(p^{i}\right)=1_{i=0} x$. En effet, si ceci est vrai et si $\left(x_{i}\right)_{i}$ est une suite presque nulle dans $X_{\infty}^{-}$, il existe pour chaque $l \in \mathbf{Z}$ un $v_{l}^{\prime} \in \Pi_{c}^{P-\text { alg }}$ tel que $\phi_{v_{l}^{\prime}}\left(p^{i}\right)=1_{i=0} x_{l}$. L'injectivité de $v \rightarrow \phi_{v}$ et le fait que $\left(x_{l}\right)_{l}$ est une suite presque nulle montrent que $\left(v_{l}^{\prime}\right)_{l}$ est une suite presque nulle. On peut donc définir

$$
v=\sum_{l \in \mathbf{Z}}\left(\begin{array}{cc}
p^{-l} & 0 \\
0 & 1
\end{array}\right) v_{l}^{\prime}
$$

et on vérifie facilement que $\phi_{v}\left(p^{i}\right)=x_{i}$.

Soit donc $x \in X_{\infty}^{-}$et soient $n \geq 1, \hat{x} \in N_{\text {dif }, n}$ dont l'image dans $X_{\infty}^{-}$est $x$. Soit $\omega=\frac{T}{\varphi^{-1}(T)} \in \tilde{\mathbf{A}}^{+}$. On va chercher $v$ de la forme $v=\tilde{z}\left(\bmod \tilde{D}^{+}\right) \in \Pi=\tilde{D} / \tilde{D}^{+}$avec $\tilde{z}=\omega^{-k} \tilde{y}$ et $\tilde{y} \in \tilde{D}^{+}$. Comme $\theta\left(\varphi^{i}(\omega)\right) \neq 0$ pour $i \neq 0$, pour un tel $\tilde{z}$ on a forcément $\varphi^{i}(\tilde{z}) \in \tilde{D}_{\text {dif }}^{+}$pour tout $i \neq 0$. Ceci combiné au fait que $\phi_{v}(a x)=\sigma_{a}\left(\phi_{v}(x)\right)$ pour $a \in \mathbf{Z}_{p}^{*}$ montre que $\operatorname{supp}\left(\phi_{v}\right) \subset \mathbf{Z}_{p}^{*}$. La condition $\phi_{v}(1)=x$ équivaut à $\tilde{y}-\omega^{k} \hat{x} \in \omega^{k} \tilde{D}_{\text {dif }}^{+}$. Comme $\omega^{k} \mathbf{B}_{\mathrm{dR}}^{+}=t^{k} \mathbf{B}_{\mathrm{dR}}^{+}$et $t^{k} N_{\mathrm{dif}, n} \subset D_{\mathrm{dif}, n}^{+} \subset \tilde{D}_{\mathrm{dif}}^{+}$, l'élément $u=\omega^{k} \hat{x}$ est dans $\tilde{D}_{\mathrm{dif}}^{+}$. On peut donc conclure quant à l'existence de $\tilde{y}$ grâce au lemme suivant :

Lemme 5.5. Pour tous $N \geq 1$ et $u \in \tilde{D}_{\text {dif }}^{+}$, il existe $\tilde{y} \in \tilde{D}^{+}$tel que $\tilde{y}-u \in \omega^{N} \tilde{D}_{\text {dif }}^{+}$.

Démonstration. On démontre le résultat par récurrence sur $N$. D'après [10, lemme V.1.7], l'application $\theta: \tilde{D}^{+} \rightarrow\left(\mathbf{C}_{p} \otimes \mathbf{Q}_{p} V\right)^{H}$ est surjective. Donc pour tout $u \in \tilde{D}_{\text {dif }}^{+}$il existe $\tilde{y} \in \tilde{D}^{+}$tel que $\theta(\tilde{y})=\theta(u)$, i.e. $\tilde{y}-u \in \omega \cdot \tilde{D}_{\text {dif }}^{+}$. Cela démontre le cas $N=1$. 
Supposons le résultat vrai pour $N$ et soit $u \in \tilde{D}_{\text {dif }}^{+}$. Il existe $\tilde{y} \in \tilde{D}^{+}$et $u_{1} \in \tilde{D}_{\text {dif }}^{+}$tel que $\tilde{y}-u=\omega^{N} \cdot u_{1}$. Soit $\tilde{y_{1}} \in \tilde{D}^{+}$tel que $\tilde{y_{1}}-u_{1} \in \omega \cdot \tilde{D}_{\text {dif }}^{+}$. Alors $\left(\tilde{y}-\omega^{N} \cdot \tilde{y_{1}}\right)-u \in \omega^{N+1} \cdot \tilde{D}_{\text {dif }}^{+}$.

Pour finir la preuve, il reste à vérifier que $v \in \Pi_{c}^{P-\text { alg }}$. Par construction, $T^{k} \tilde{z} \in \tilde{D}^{+}$, donc $v$ est tué par $\left(\left(\begin{array}{ll}1 & 1 \\ 0 & 1\end{array}\right)-1\right)^{k}$, donc $v \in \Pi^{U-\text { alg. De plus, comme }}$

$$
\prod_{j=0}^{k-1}\left(\sigma_{1+p^{n}}-\left(1+p^{n}\right)^{i}\right) N_{\mathrm{dif}, n} \subset D_{\mathrm{dif}, n}^{+}
$$

(cela est immédiat, voir par exemple [10, lemme VI.4.1]) et comme $\operatorname{Im}\left(\phi_{v}\right) \subset N_{\text {dif }, n} / D_{\text {dif }, n}^{+}$, on a $\phi_{\lambda_{n} v}=0$ (cf. définition 4.9 pour $\lambda_{n}$ ) et donc $\lambda_{n} v=0$ et $v \in \Pi^{P-a l g}$. Par construction, $\operatorname{Im}\left(\phi_{v}\right)$ est compact dans $\mathbf{Q}_{p}^{*}$, d'où le résultat.

\subsection{Vecteurs presque algébriques et représentations de de Rham}

On démontre dans cette partie le théorème 1.5. Cela utilise essentiellement tous les résultats des chapitres précédents. On suppose que $V$ est à poids de Hodge-Tate 0 et $k \in \mathbf{N}^{*}$ (d'après la proposition [5.3, à un twist près ces hypothèses sont nécessaires si

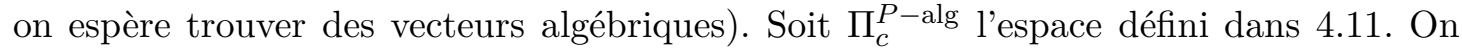
commence par démontrer la caractérisation suivante des représentations de de Rham.

Théorème 5.6. $V$ est de de Rham si et seulement si $\left(u^{-}\right)^{k}$ tue $\Pi_{c}^{P-\text { alg }}$.

Démonstration. Par dualité, $\left(u^{-}\right)^{k}$ tue $\Pi_{c}^{P-\text { alg }}$ si et seulement si $\left(u^{-}\right)^{k}\left(\check{\Pi}^{\text {an }}\right)^{*}$ est orthogonal à $\Pi_{c}^{P-\text { alg }}$ pour l'accouplement $[,]_{\mathbf{P}^{1}}$ (voir 4.3). La proposition 4.12 combinée à la proposition 5.4 et au lemme 4.7 montre que ceci se passe si et seulement si $i_{l}^{+}\left(\left(u^{-}\right)^{k} z\right)=0$ dans $\lim _{\longleftarrow} D_{\mathrm{dif}, n}^{+} / t^{k} N_{\mathrm{dif}, n}$, pour tout $l \in \mathbf{Z}$ et pour tout $z \in\left(\check{\Pi}^{\text {an }}\right)^{*}$, ou encore $i_{l, n}\left(\left(u^{-}\right)^{k} z\right) \in t^{k} \overleftarrow{N}_{\text {dif }, n}$ pour tout $n \geq m(D), l \in \mathbf{Z}$ et $z \in\left(\check{\Pi}^{\text {an }}\right)^{*}$. Par définition des $i_{l, n}$, cela arrive si et seulement si $\varphi^{-n}\left(\left(u^{-}\right)^{k} \operatorname{Res}_{\mathbf{Z}_{p}}(z)\right) \in t^{k} N_{\text {dif }, n}$ pour tout $n \geq m(D)$ et tout $z \in\left(\check{\Pi}^{\text {an }}\right)^{*}$. Nous aurons besoin du résultat suivant :

Lemme 5.7. Pour tout $z \in D_{\text {rig }}$ et tout $j \geq 1$ on a

$$
\left(u^{-}\right)^{j}(z)=\frac{\nabla(\nabla-1) \ldots(\nabla-j+1)(k-\nabla)(k+1-\nabla) \ldots(k+j-1-\nabla)(z)}{t^{j}} .
$$

Démonstration. Ceci découle par une récurrence immédiate du théorème 1.1, en utilisant la formule

$$
\nabla(k-\nabla)\left(\frac{x}{t^{j}}\right)=\frac{(k+j-\nabla)(\nabla-j)(x)}{t^{j}},
$$

qui se vérifie par un calcul direct. 
La conclusion du lemme 5.7 (avec $j=k$ ) et du premier paragraphe est que $\left(u^{-}\right)^{k}$ tue $\Pi_{c}^{P-\text { alg }}$ si et seulement si $\varphi^{-n}\left(\operatorname{Res}_{\mathbf{Z}_{p}}(z)\right) \in X_{n}$ (voir le lemme 4.4 pour la définition de $\left.X_{n}\right)$ pour tout $z \in\left(\check{\Pi}^{\text {an }}\right)^{*}$ et tout $n \geq m(D)$. La partie a) du lemme 4.4 permet de conclure directement dans le cas de Rham.

Supposons donc que $V$ n'est pas de de Rham et que $\left(u^{-}\right)^{k}$ tue $\Pi_{c}^{P-\text { alg }}$. Soit $z_{1} \in D^{\psi=1}$. La proposition V.2.1 de [10] (plutôt sa preuve) montre qu'il existe $z \in\left(\check{\Pi}^{\text {an }}\right)^{*}$ tel que $\operatorname{Res}_{\mathbf{Z}_{p}}(z)=z_{1}$. On a donc $\varphi^{-n}\left(z_{1}\right) \in X_{n}$. Mais $D$ a une base $z_{1}, z_{2}$ sur $\mathscr{E}$ formée d'éléments de $D^{\psi=1}$ (voir [7, corollaire I.7.6]), et $z_{1}, z_{2}$ forment aussi une base de $D^{\dagger}$ sur $\mathscr{E}^{\dagger}$ (car $\mathscr{E}^{\dagger}$ est un corps), donc de $D_{\text {rig }}$ sur $\mathscr{R}$. On en déduit que pour tout $23 n \geq m(D)$, $z_{1}$ et $z_{2}$ forment une base de $D^{\left[0, r_{n}\right]}$ sur $\mathscr{E}^{\left[0, r_{n}\right]}$. Comme $\varphi^{-n}\left(z_{1}\right)$ et $\varphi^{-n}\left(z_{2}\right) \in X_{n}$ et comme $X_{n}$ est un $L_{n}[[t]]$-module, on obtient $\varphi^{-n}\left(D^{\left.] 0, r_{n}\right]}\right) \subset X_{n}$ et donc $D_{\text {dif }, n}^{+} \subset X_{n}$. Mais cela contredit le point b) du lemme 4.4. Cela permet de conclure.

La preuve du théorème suivant (c'est le théorème 1.5 de l'introduction) est maintenant une formalité.

Théorème 5.8. $V$ est de de Rham à poids de Hodge-Tate distincts si et seulement si $\Pi$ a des vecteurs localement algébriques non nuls.

Démonstration. Quitte à faire une torsion par un caractère localement algébrique, on peut supposer qu'un des poids de Hodge-Tate de $V$ est 0 . La proposition 5.3 permet de supposer que l'autre poids de Hodge-Tate est $k$, un entier strictement positif.

Supposons que $V$ n'est pas de de Rham, mais que $\Pi^{\text {alg }} \neq 0$. D'aprè 24 [10, cor. VI.5.9, on a $\Pi_{c}^{P-\text { alg }} \subset \Pi^{\text {alg }}$ et d'après la proposition $\left[5.3\right.$, on sait que $\Pi^{\text {alg }}=\operatorname{Sym}^{k-1} \otimes \Pi^{\text {lc }}$ pour une représentation lisse $\Pi^{\mathrm{lc}}$. En particulier, $\left(u^{-}\right)^{k}$ tue $\Pi_{c}^{P-\text { alg }}$, contradiction avec le théorème 5.6 .

Supposons que $V$ est de de Rham. Fixons $n>m(D)$ et soit $e_{1}, e_{2}$ comme dans la proposition 4.1 (c'est une base de $D_{\mathrm{dR}}(V)$ ). D'après la proposition [5.4, il existe $v \in$ $\Pi_{c}^{P-\text { alg }}$ tel que

$$
\phi_{v}\left(p^{i}\right)=1_{i=0} t^{k-1} e_{2} \quad\left(\bmod D_{\mathrm{dif}, n}^{+}\right) .
$$

$\phi_{v}$ est alors à support dans $\mathbf{Z}_{p}^{*}$ (prop 4.10) et, si on pose $u=\left(\begin{array}{ll}1 & 1 \\ 0 & 1\end{array}\right)$, on a (en utilisant l'égalité $\left[(1+T)^{x}\right]=(1+T)^{x}=e^{t x}$ pour $\left.x \in \mathbf{Z}_{p}\right)$

$$
\phi_{(u-1) v}(x)=\left(\left[(1+T)^{x}\right]-1\right) \phi_{v}(x)=1_{x \in \mathbf{Z}_{p}^{*}}\left(e^{t x}-1\right) \phi_{v}(x)=0 \quad\left(\bmod D_{\mathrm{dif}, n}^{+}\right),
$$

donc $u v=v$, i.e. $v$ est invariant $\operatorname{par}\left(\begin{array}{cc}1 & \mathbf{Z}_{p} \\ 0 & 1\end{array}\right)$. Si $a_{n}^{+}=\left(\begin{array}{cc}1+p^{n} & 0 \\ 0 & 1\end{array}\right)$, alors $a_{n}^{+} v=\left(1+p^{n}\right)^{k-1} v$, car pour tout $x \in \mathbf{Q}_{p}^{*}$ on a

$$
\begin{aligned}
& \phi_{a_{n}^{+} v-\left(1+p^{n}\right)^{k-1} v}(x)=\phi_{v}\left(\left(1+p^{n}\right) x\right)-\left(1+p^{n}\right)^{k-1} \phi_{v}(x)= \\
& 1_{x \in \mathbf{Z}_{p}^{*}} \sigma_{x}\left(\sigma_{1+p^{n}}-\left(1+p^{n}\right)^{k-1}\right)\left(t^{k-1} e_{2}\right)=0 \quad\left(\bmod D_{\mathrm{dif}, n}^{+}\right) .
\end{aligned}
$$

\footnotetext{
${ }^{23}$ Cela peut demander d'augmenter $m(D)$.

${ }^{24}$ C'est une conséquence de l'irréductibilité sous l'action du sous-groupe de Borel de l'espace des fonctions localement constantes à support compact dans $\mathbf{Q}_{p}^{*}$, voir le lemme 2.9.1 de [25].
} 
Enfin, notons que $v \neq 0$, car $\phi_{v}(1)=t^{k-1} e_{2} \neq 0\left(\bmod D_{\text {dif }, n}^{+}\right)$.

D'après la proposition 4.12, on a $v \in \Pi^{\text {an }}$ et le paragraphe précédent montre que $u^{+}(v)=0$ et $h(v)=(k-1) v$. On va montrer que $v$ est localement algébrique. D'après la proposition 5.2, il suffit de montrer la $\mathfrak{g l}_{2}$-équivariance du morphisme $L$-linéaire $f$ : $\operatorname{Sym}^{k-1}\left(L^{2}\right) \rightarrow \Pi^{\text {an }}$, défini par

$$
f\left(e_{1}^{k-j-1} e_{2}^{j}\right)=\frac{\left(u^{-}\right)^{j} v}{(k-1)(k-2) \ldots(k-j)}
$$

pour $0 \leq j \leq k-1$.

Si $x_{j}=e_{1}^{k-j-1} e_{2}^{j}$, un calcul immédiat montre que

$$
h\left(x_{j}\right)=(k-2 j-1) x_{j}, \quad u^{+}\left(x_{j}\right)=j x_{j-1}, \quad u^{-}\left(x_{j}\right)=(k-1-j) x_{j+1},
$$

avec, par convention, $x_{-1}=x_{k}=0$. Soit $y_{j}=\frac{\left(u^{-}\right)^{j} v}{(k-1)(k-2) \ldots(k-j)}$. On veut montrer que les $y_{j}$ satisfont les mêmes relations. Mais les relations

$$
\left[u^{+}, u^{-}\right]=h, \quad\left[h, u^{+}\right]=2 u^{+}, \quad\left[h, u^{-}\right]=-2 u^{-}
$$

et une récurrence immédiate montrent que, dans $U\left(\mathfrak{g l}_{2}\right)$,

$$
\left(u^{-}\right)^{j} h=(h+2 j)\left(u^{-}\right)^{j}, \quad u^{+}\left(u^{-}\right)^{j}=\left(u^{-}\right)^{j} u^{+}+j\left(u^{-}\right)^{j-1}(h-j+1) .
$$

La première et le fait que $h v=(k-1) v$ entraînent $h\left(y_{j}\right)=(k-1-2 j) y_{j}$. La seconde et la relation $u^{+} v=0$ donnent $u^{+}\left(y_{j}\right)=j y_{j-1}$. Enfin, il est clair que $u^{-}\left(y_{j}\right)=(k-1-j) y_{j+1}$ si $j<k-1$ et tout le point est de le vérifier pour $j=k-1$, i.e. que $\left(u^{-}\right)^{k} v=0$. Mais cela découle du théorème 5.6 et finit la preuve du théorème.

\section{Références}

[1] K. Ardakov, S.J. Wadsley, On irreducible representations of compact p-adic analytic groups, preprint, disponible à http://www.maths.nottingham.ac.uk/personal/pmzka1/.

[2] L. Berger-Représentations p-adiques et équations différentielles, Invent. Math. 148 (2002), p.219284.

[3] L. Berger, C. Breuil-Sur quelques représentations potentiellement cristallines de $G_{\mathbf{Q}_{p}}$, Astérisque 330 , p. $155-211$.

[4] L. Berger, P. Colmez-Familles de représentations de de Rham et monodromie p-adique, Astérisque 319(2008), p. 303-337.

[5] C. Breuil-Invariant $L$ et série spéciale p-adique, Ann. Sci. Ecole Norm. Sup 37 (2004), p. 559-610.

[6] F. Cherbonnier, P. Colmez-Représentations p-adiques surconvergentes, Invent. Math. 133 (1998), p. 581-611.

[7] F. Cherbonnier, P. Colmez-Théorie d'Iwasawa des représentations p-adiques d'un corps local, J.A.M.S, Volume 12, Number 1, January 1999, Pages 241-268. 
[8] P. Colmez- Théorie d'Iwasawa des représentations de de Rham d'un corps local, Ann. of Math. 148 (1998), p. 485-571.

[9] P. Colmez- $(\varphi, \Gamma)$-modules et représentations du mirabolique de $\mathrm{GL}_{2}\left(\mathbf{Q}_{p}\right)$, Astérisque 330 (2010), p. 61-153.

[10] P. Colmez-Représentations de $\mathrm{GL}_{2}\left(\mathbf{Q}_{p}\right)$ et $(\varphi, \Gamma)$-modules, Astérisque 330 (2010), p. 281-509.

[11] P. Colmez-La série principale unitaire de $\mathrm{GL}_{2}\left(\mathbf{Q}_{p}\right)$, Astérisque 330, p.213-262.

[12] P. Colmez-La série principale unitaire de $\mathrm{GL}_{2}\left(\mathbf{Q}_{p}\right)$ : vecteurs localement analytiques, en préparation.

[13] P. Colmez-Espaces Vectoriels de dimension finie et représentations de de Rham, Astérisque 319 (2008), p.117-186.

[14] P. Colmez-Les conjectures de monodromie p-adiques, Sém. Bourbaki 2001-02, exp. 897, Astérisque 290 (2003), p.53-101.

[15] G. Dospinescu-Equations différentielles $p$-adiques et foncteurs de Jacquet analytiques, appendice à 12 , en préparation.

[16] G. Dospinescu, B. Schraen, Endomorphism algebras of admissible $p$-adic representations of $p$-adic Lie groups, preprint, disponible à http://arxiv.org/abs/1106.2446.

[17] G. Dospinescu, Sur l'image du foncteur de Colmez, en préparation.

[18] M. Emerton-A local-global compatibility conjecture in the $p$-adic Langlands programme for $G L_{2} / \mathbf{Q}$, Pure Appl. Math. Q. 2 (2006), p. 279-393.

[19] M. Emerton-Local-global compatibility in the $p$-adic Langlands programme for $G L_{2} / \mathbf{Q}$, preprint, disponible à http://www.math.northwestern.edu/ emerton/preprints.html.

[20] M. Emerton-Locally analytic vectors in representations of locally padic analytic groups, to appear in Memoirs of the AMS, disponible à http://www.math.northwestern.edu/ emerton/preprints.html

[21] M. Emerton-On the interpolation of systems of eigenvalues attached to automorphic Hecke eigenforms, Invent. Math. 164 (2006), no. 1, 1-84.

[22] J.-M. Fontaine-Sur Certains Types de Représentations p-Adiques du Groupe de Galois d'un Corps Local ; Construction d'un Anneau de Barsotti-Tate, Ann. of Math. 115 (1982), p.529-577.

[23] J.-M. Fontaine-Représentations p-adiques des corps locaux. I, in The Grothendieck Festschrift, Vol II, Progr. Math., vol 87, Birkhauser, 1990, p. 249-309.

[24] J.-M. Fontaine-Arithmétique des représentations galoisiennes p-adiques, Astérisque 295 (2004), p. $1-115$.

[25] H. Jacquet, R. Langlands-Automorphic forms on GL(2), Springer Lecture Notes in Mathematics, 1970, pp. 1-548.

[26] K. Kato-Lectures on the approach to Iwasawa theory for Hasse-Weil L-functions via $B_{d R}$. I, in Arithmetic algebraic geometry (Trento, 1991), Lecture notes in Math., vol.1553, Springer, 1993, p.50-163.

[27] K.S. Kedlaya-A p-adic local monodromy theorem, Ann. of Math. 160 (2004), p.93-184. 
[28] M. Kisin-Deformations of $G_{\mathbf{Q}_{p}}$ and $\mathrm{GL}_{2}\left(\mathbf{Q}_{p}\right)$ representations, Astérisque 330, 2010, p. 511-528.

[29] M. Kisin-The Fontaine-Mazur conjecture for $G L_{2}$, J. Amer. Math. Soc 22(3)(2009), p. 641-690.

[30] V. Paškūnas-The image of Colmez's Montréal functor, preprint, disponible à http://arxiv.org/abs/1005.2008

[31] B. Perrin-Riou-Théorie d'Iwasawa des représentations $p$-adiques sur un corps local, Invent. Math. 115 (1994), p.81-161.

[32] S. Sen-Continuous cohomology and p-adic Galois representations, Invent. Math 62 (1980/1981), p. 89-116.

[33] P. Schneider, J. Teitelbaum- $U(\mathfrak{g})$-finite locally analytic representations, Represent. Theory 5 (2001), p 111-128.

[34] P. Schneider, J. Teitelbaum- Locally analytic distributions and $p$-adic representation theory, with applications to $G L_{2}$, J. Amer. Math. Soc 15(2002), p. 443-468.

[35] P. Schneider, J. Teitelbaum- Algebras of $p$-adic distributions and admissible representations, Invent. Math. 153 (2003), p. 145-196. 